Comment. Math. Helv. 76 (2001) 436-466

(C) 2001 Birkhäuser Verlag, Basel

Commentarii Mathematici Helvetici

\title{
Deformation and Cohen-Macaulayness of the multicone over the flag variety
}

\author{
Rocco Chirivì
}

\begin{abstract}
A general theory of LS algebras over a multiposet is developed. As a main result, the existence of a flat deformation to discrete algebras is obtained. This is applied to the multicone over partial flag varieties for Kac-Moody groups proving a deformation theorem to a union of toric varieties. In order to achieve the Cohen-Macaulayness of the multicone we show that Bruhat posets (defined as glueing of minimal representatives modulo parabolic subgroups of a Weyl group) are lexicographically shellable.
\end{abstract}

Mathematics Subject Classification (2000). 14M15, 06A07.

Keywords. Multicone, flag variety, standard monomial theory, Bruhat order, shellability.

\section{Introduction}

This paper is a continuation of [5]. The main purpose is to set up the combinatorial and algebraic machinery to treat the multicone over (partial) flag varieties for KacMoody groups. In order to do this some generalizations of LS algebras are needed, but the general framework remains the same.

First some combinatorics is developed. We begin introducing the notion of multiposet, a "glueing" of posets, as follows. Suppose we are given $n$ posets with bonds $\left(\mathrm{S}_{i}, \leq_{i}, f_{i}\right)$, and liftings $\leq_{i, j}$ of $\left(\mathrm{S}_{i}, \leq_{i}\right),\left(\mathrm{S}_{j}, \leq_{j}\right)$ to the disjoint union $\mathrm{S}_{i} \sqcup \mathrm{S}_{j}$. We define the sets $\mathrm{L}_{2}^{i, j}$ as the set of pairs of LS paths $\left(\ell, \ell^{\prime}\right) \in$ $\mathrm{L}_{1}\left(\mathrm{~S}_{i}\right) \times \mathrm{L}_{1}\left(\mathrm{~S}_{j}\right)$ such that $\max \operatorname{supp} \ell \leq_{i, j} \min \operatorname{supp} \ell^{\prime}$. Suppose moreover that we are given maps $\phi_{i, j}: \mathrm{L}_{2}^{i, j} \rightarrow \mathrm{L}_{2}^{j, i}$ (satisfying some mild conditions). We call the data $\left(\mathrm{S}_{i}, \leq_{i}, f_{i}, \leq_{i, j}, \phi_{i, j}\right)$ a multiposet with bonds and we call the maps $\phi_{i, j}$ the swappings.

Then we see the notions of LS paths, weak standard and standard monomials for a multiposet. We note here that at this point we allow our posets to be infinite (this is mandatory since $\mathrm{W} / \mathrm{W}_{\mathrm{P}}$, where $\mathrm{W}$ is the Weyl group of some Kac-Moody group $\mathrm{G}$ and $\mathrm{P}$ is some parabolic subgroup, is in general infinite) but we ask for

\footnotetext{
The author was partially supported by a grant of the Ateneo of Rome "La Sapienza".
} 
some "lower finiteness condition". See Section 1.

The algebraic side of the machinery is treated in Section 2 and Section 3. An LS algebra over a multiposet is defined as an algebra $\mathrm{A}$, over some base ring $\mathrm{R}$, having as $\mathrm{R}$-basis the set of standard monomials and satisfying the property that when a monomial is expressed in terms of standard monomials then only standard monomials satisfying some lexicographic condition do appear. Then, in analogy with [5], we construct a general theory of flat deformations to discrete algebras and we give a result about Cohen-Macaulayness.

In Sections 4 and 5 we give our application to the multicone. At this point our main sources are the plactic algebras (see [19]) and the standard monomial theory (see [16], [18] and [20]). Notice that these works are slightly extended: some results about the good string property (see [19] and Definition 5.1) of parabolic subgroups is generalized to Kac-Moody groups and some relations of the standard monomial theory for LS paths of different shapes are needed.

Our main results are the following. Let $\mathrm{G}$ be a Kac-Moody group and let $\mathrm{P}$ be a parabolic subgroup. Then the flag variety $\mathrm{G} / \mathrm{P}$ admits a flat deformation to a union of sections of toric varieties $X$ (Theorem 4.1). If $\mathrm{P}$ has the good string property then $X$ is simply the union of toric varieties (without sections). A criterion for a parabolic subgroup to have the good string property is also given: this result is very neat for the finite and affine types. In the good string case we obtain a new proof of the fundamental result in [12], i.e. the quadratic relations suffice to generate the relations in $\oplus_{a_{1}, \ldots, a_{n} \geq 0} H^{0}\left(\mathrm{G} / \mathrm{B}, \mathcal{L}_{a_{1} \lambda_{1}+\cdots+a_{n} \lambda_{n}}\right)$, where $\mathcal{L}_{\lambda}$ is the line bundle $\mathrm{G} \times_{\mathrm{B}} k_{-\lambda}$ over $\mathrm{G} / \mathrm{B}$. Finally we prove that in the good string case the multicone is Cohen-Macaulay for $\mathrm{G}$ of finite type. To achieve this result one needs the Cohen-Macaulayness of the involved posets. We devote the last four sections to this goal; however a more general result is obtained. Let us explain this in details.

Let $(\mathrm{W}, \mathrm{S})$ be a Coxeter group and let $\mathrm{I}, \mathrm{J}$ be subsets of $\mathrm{S}$. The sets $\mathrm{W}^{\mathrm{I}}, \mathrm{W}^{\mathrm{J}}$ of minimal representatives modulo the parabolic subgroups $\mathrm{W}_{\mathrm{I}}, \mathrm{W}_{\mathrm{J}}$ inherit the Bruhat order by restriction from $\mathrm{W}$. Consider now the disjoint union $\mathrm{W}^{(\mathrm{I}, \mathrm{J})}=$ $\mathrm{W}^{\mathrm{I}} \sqcup \mathrm{W}^{\mathrm{J}}$. $\mathrm{W}^{(\mathrm{I}, J)}$ has a Bruhat order extending that of $\mathrm{W}^{\mathrm{I}}, \mathrm{W}^{\mathrm{J}} \hookrightarrow \mathrm{W}^{(\mathrm{I}, J)}$. We call this poset structure the Bruhat poset $\mathrm{W}^{\Omega}$ where $\Omega=(\mathrm{I}, \mathrm{J})$. One may clearly generalize this contruction to $\Omega=\left(\mathrm{I}_{1}, \ldots, \mathrm{I}_{n}\right)$ considering various parabolic subgroups $\mathrm{W}_{\mathrm{I}_{1}}, \ldots, \mathrm{W}_{\mathrm{I}_{n}}$ associated to subsets $\mathrm{I}_{1}, \ldots, \mathrm{I}_{n} \subset \mathrm{S}$.

Assume now that $\mathrm{W}$ is finite. In this case the adjacency relation $\tau \triangleleft \sigma$ in $\mathrm{W}^{\Omega}$ for $\tau \in \mathrm{W}^{\mathrm{I}_{h}}, \sigma \in \mathrm{W}^{\mathrm{I}_{h+1}}$ relative to the order defined above, has a sharp description: there exists $w \in \mathrm{W}$, depending on $\mathrm{I}_{h}, \mathrm{I}_{h+1}$, such that $\tau \triangleleft \sigma$ if and only if $\tau=\sigma w$. This is the key result one needs in order to extend the lexicographic labeling introduced in [2] to the Bruhat poset $\mathrm{W}^{\Omega}$. Our main result is then: any closed interval $[\tau, \sigma] \subset \mathrm{W}^{\Omega}$ is lexicographically shellable. We apply this to obtain that $k\left\{\mathrm{~W}^{\Omega}\right\}$, the Stanley-Reisner ring of $\mathrm{W}^{\Omega}$, is Cohen-Macaulay, using a well known theorem (see [21]).

These results are mainly a generalization of the method of [2]: the proof of the 
shellablity in $\mathrm{W}^{\Omega}$ is an adaptation of Björner and Wachs's proof of shellabilty in $\mathrm{W}^{\mathrm{I}}$, taking into account the description of adjacency relation in $\mathrm{W}^{\Omega}$. Shellability of $\mathrm{W}^{\Omega}$ had already been proved in [10] in the following special cases: $\mathrm{W}$ of type $\mathrm{A}_{\ell}, \mathrm{B}_{\ell}, \mathrm{C}_{\ell}, \Omega=\left(\mathrm{I}_{1}, \ldots, \mathrm{I}_{\ell}\right)$ where $\mathrm{I}_{h}=\mathrm{S} \backslash\left\{s_{h}\right\}, h=1, \ldots, \ell$, i.e. the $\mathrm{W}_{\mathrm{I}_{h}}$ are the maximal parabolic subgroups and they are ordered naturally in the same way as the corresponding omitted roots in the Dynkin diagram are. The proof of this in [10] is a case by case analysis and heavily depends on combinatorial descriptions of minimal representatives modulo maximal parabolic subgroups.

Section 6 introduces Coxeter groups, parabolic subgroups and Bruhat order. The main related results are provided for easier reference. In Section 7 we define the Bruhat poset as a "glueing" of minimal representative sets. In the case of finite $\mathrm{W}$ we prove a sort of "homogeneity" for the adjacency relation in $\mathrm{W}^{\Omega}$. The last half of Section 7 is devoted to the proof that any Bruhat poset is graded. Lexicographic shellable posets are introduced in Section 8. This section is just a short form of Section 2 in [2]. Finally Section 9 describes our labeling of the intervals $[\tau, \sigma] \subset \mathrm{W}^{\Omega}$, proving that this is actually an L-labeling.

I would like to express my thanks to Prof. P. Littelmann, Prof. C. De Concini and Prof. A. Björner for many useful conversations. I also wish to thank Prof. C.S. Sheshadri who suggested me to study the multicone. Finally it is a pleasure to thank Prof. A. D'Andrea for improvements to the exposition.

\section{Multiposet and standard monomials}

We begin this section by briefly recalling the definitions of poset with bonds, LS paths, etc. We refer to [5] for details and proofs.

Let $(\mathrm{S}, \leq)$ be a poset. In this paper poset means partially ordered set with the following finiteness property: for every $a \in \mathrm{S}$ the set $\{b \in \mathrm{S} \mid b \leq a\}$ is finite. Let $f: \tilde{\mathrm{S}} \longrightarrow \mathbb{N}$ be a map on the set $\tilde{\mathrm{S}}$ of all adjacent pairs $a<b$ in $\mathrm{S}$, such that $\operatorname{gcd}\left\{f\left(a_{i}, a_{i+1}\right) \mid i=1, \ldots, s-1\right\}=\operatorname{gcd}\left\{f\left(b_{j}, b_{j+1}\right) \mid j=1, \ldots, t-1\right\}$ for any pair of complete chains $a=a_{1}<\ldots<a_{s}=b, a=b_{1}<\ldots<b_{t}=b$ from $a$ to $b$ in $\mathrm{S}$. We call the data $(\mathrm{S}, \leq, f)$ a poset with bonds. Notice that we can extend the map $f$ to all pairs $a<b$ setting $f(a, b)=\operatorname{gcd}\left\{f\left(a_{i}, a_{i+1}\right) \mid i=1, \ldots, s-1\right\}$ for a complete chain $a=a_{1}<\ldots<a_{s}=b$.

Given a poset with bonds we define an $L S$ path of degree $r \in \mathbb{N}$ as a pair

$$
\ell=\left(a_{1}<\cdots<a_{s} ; 0=x_{0}<x_{1}<\cdots<x_{s}=r\right)
$$

of finite linearly ordered elements of $\mathrm{S} a_{1}<\cdots<a_{s}$ and rational numbers $0=x_{0}<x_{1}<\cdots<x_{s}=r$ such that $x_{i} f\left(a_{i}, a_{i+1}\right) \in \mathbb{Z}$ for any $i=1, \ldots, s-1$. Let $\mathrm{L}_{r}(\mathrm{~S})$ be the set of all LS paths of degree $r$ and let $\mathrm{L}(\mathrm{S})=\cup_{r} \mathrm{~L}_{r}(\mathrm{~S})$.

We associate with any LS path $\ell=\left(a_{1}<\cdots<a_{s} ; 0=x_{0}<x_{1}<\cdots<x_{s}=r\right)$ a map $\gamma_{\ell}: \mathrm{S} \longrightarrow \mathbb{N}$ defined as $\gamma_{\ell}(a)=0$ if $a \notin \operatorname{supp} \ell=\left\{a_{1}, \ldots, a_{s}\right\}$ and $\gamma_{\ell}\left(a_{i}\right)=N_{a_{i}}\left(x_{i}-x_{i-1}\right)$ where $N_{a}=\operatorname{lcm}\{f(x, y) \mid x=a$ or $y=a\}$. Given two 
LS paths $\ell_{1}, \ell_{2}$ with compatible support, i.e. such that $\operatorname{supp} \ell_{1} \cup \operatorname{supp} \ell_{2} \subset \mathrm{C}$ for some chain $\mathrm{C}$ in $\mathrm{S}$, it is easy to see that there exists a unique LS path $\ell$ such that $\gamma_{\ell}=\gamma_{\ell_{1}}+\gamma_{\ell_{2}}$, and we denote it by $\ell_{1} \circ \ell_{2}$. Moreover the support of $\ell=\ell_{1} \circ \ell_{2}$ is contained in C. If $\ell_{1}, \ell_{2}$ are two LS paths whose support is not compatible then we write $\ell_{1} \circ \ell_{2} \notin \mathrm{L}(\mathrm{S})$. Further we have

Proposition 1.1. If $\ell$ is an LS path of degree $r$, then there exist uniquely defined $L S$ paths of degree one $\ell_{1}, \ldots, \ell_{r}$ such that $\max \operatorname{supp} \ell_{i} \leq \min \operatorname{supp} \ell_{i+1}$ for $i=$ $1, \ldots, r-1$, and $\ell=\ell_{1} \circ \cdots \circ \ell_{r}$.

We will refer to $\ell_{1} \circ \cdots \circ \ell_{r}$ as the canonical decomposition of $\ell$.

Now we want to consider a more general framework. Suppose we are given $n$ posets with bonds

$$
\left(\mathrm{S}_{1}, \leq_{1}, f_{1}\right),\left(\mathrm{S}_{2}, \leq_{2}, f_{2}\right), \ldots,\left(\mathrm{S}_{n}, \leq_{n}, f_{n}\right)
$$

and for each $1 \leq i, j \leq n$ a lifting of $\left(\mathrm{S}_{i}, \leq_{i}\right)$ and $\left(\mathrm{S}_{j}, \leq_{j}\right)$ to a poset $\left(\mathrm{S}_{i} \sqcup\right.$ $\left.\mathrm{S}_{j}, \leq_{i, j}\right)$. Let $\mathrm{L}_{2}^{i, j}$ denote the subset of $\mathrm{L}_{1}\left(\mathrm{~S}_{i}\right) \times \mathrm{L}_{1}\left(\mathrm{~S}_{j}\right)$ of pairs $\left(\ell, \ell^{\prime}\right)$ such that $\max \operatorname{supp} \ell \leq_{i, j} \min \operatorname{supp} \ell^{\prime}$. Finally suppose that we are given bijections $\phi_{i, j}: \mathrm{L}_{2}^{i, j} \longrightarrow \mathrm{L}_{2}^{j, i}$ such that $\phi_{i i}=\operatorname{Id}_{\mathrm{L}_{1}\left(\mathrm{~S}_{i}\right) \times \mathrm{L}_{1}\left(\mathrm{~S}_{i}\right)}$ and $\phi_{j, i}=\phi_{i, j}^{-1}$. We refer to such maps as swappings. We call the data of the posets with bonds, liftings and swappings a multiposet with bonds

$$
\mathrm{S}=\left(\mathrm{S}_{i}, \leq_{i}, f_{i}, \leq_{i, j}, \phi_{i, j}\right) .
$$

Now we consider formal monomials of LS paths, i.e. monomials $\ell_{1} \cdots \ell_{r}$ with $\ell_{i} \in \mathrm{L}_{1}(\mathrm{~S})=\sqcup_{k=1, \ldots, n} \mathrm{~L}_{1}\left(\mathrm{~S}_{k}\right)$, and we want to define weak standard and standard LS monomials for a multiposet.

Let $\mathrm{M}_{r}$ be the set of the (formal) LS monomials $\ell_{1} \cdots \ell_{r}$, such that $\ell_{i} \in$ $\mathrm{L}_{1}\left(\mathrm{~S}_{k_{i}}, \leq_{k_{i}}, f_{k_{i}}\right)$ and maxsupp $\ell_{i} \leq_{k_{i}, k_{i+1}} \min \operatorname{supp} \ell_{i+1}$ for any $1 \leq i<r$, we call such monomials weak standard. Consider now the group $\Phi_{r}$ with generators $\tau_{1}, \ldots, \tau_{r-1}$ and relations $\tau_{1}^{2}=\cdots=\tau_{r-1}^{2}=1$. We define an action of $\Phi_{r}$ on $\mathrm{L}_{1}(\mathrm{~S}) \times \cdots \times \mathrm{L}_{1}(\mathrm{~S})(r$ times $)$ as follows:

$$
\tau_{i}\left(\ell_{1}, \ldots, \ell_{r}\right)=\left(\ell_{1}, \ldots, \ell_{i-1}, \ell_{i}^{\prime}, \ell_{i+1}^{\prime}, \ell_{i+2}, \ldots, \ell_{r}\right)
$$

where $\ell_{i} \in \mathrm{L}_{1}\left(\mathrm{~S}_{k_{i}}\right)$ and $\left(\ell_{i}^{\prime}, \ell_{i+1}^{\prime}\right)=\phi_{k_{i}, k_{i+1}}\left(\ell_{i}, \ell_{i+1}\right)$ if $\left(\ell_{i}, \ell_{i+1}\right) \in \mathrm{L}_{2}^{k_{i}, k_{i+1}}$ and $\left(\ell_{i}^{\prime}, \ell_{i+1}^{\prime}\right)=\left(\ell_{i}, \ell_{i+1}\right)$ otherwise. We call a monomial $\ell_{1} \cdots \ell_{r} \in \mathrm{M}_{r}$ standard if every monomial $\ell_{1}^{\prime} \cdots \ell_{r}^{\prime}$ in the orbit $\Phi_{r}\left(\ell_{1}, \ldots, \ell_{r}\right)$, is weak standard.

We define the multidegree of a LS monomial $\ell_{1} \cdots \ell_{r}$ as $\mathbf{a}=\left(a_{1}, \cdots, a_{n}\right) \in \mathbb{N}^{n}$ where $a_{1}$ is the number of LS paths in $\mathrm{L}_{1}\left(\mathrm{~S}_{1}\right), a_{2}$ is the number of LS paths in $\mathrm{L}_{1}\left(\mathrm{~S}_{2}\right)$, and so on. Let us set some more notation: we denote the set of all monomials with multidegree $\mathbf{a}=\left(a_{1}, \cdots, a_{n}\right)$ by $\mathrm{M}_{\mathbf{a}}$, the set of weak standard LS monomials by $\mathrm{M}^{w}$ and the set of standard LS monomials by $\mathrm{M}^{s}$, we define also $\mathrm{M}_{\mathbf{a}}^{w}=\mathrm{M}^{w} \cap \mathrm{M}_{\mathbf{a}}, \mathrm{M}_{\mathbf{a}}^{s}=\mathrm{M}^{s} \cap \mathrm{M}_{\mathbf{a}}$, and in the same way we define $\mathrm{M}_{r}^{w}$ and $\mathrm{M}_{r}^{s}$ considering the total degree $r=|\mathbf{a}|=a_{1}+\cdots+a_{n}$. 
We call a multiposet $\mathrm{S}=\left(\mathrm{S}_{i}, \leq_{i}, f_{i}, \leq_{i, j}, \phi_{i, j}\right)$ canonical if every weak standard monomial is standard, i.e. if the two notions of standardness coincide. Obviously if $n=2$ (two posets) then $\mathrm{S}$ is canonical. The numbering $\mathrm{S}_{1}, \ldots, \mathrm{S}_{n}$ is clearly unessential for standard LS monomial but, in general, weak standard monomials do depend on this numbering.

Notice that if $\mathrm{S}$ is canonical then, lifting $\left(\mathrm{S}_{1}, \leq_{1}\right), \ldots,\left(\mathrm{S}_{n} \leq_{n}\right)$ to $(\overline{\mathrm{S}}=$ $\left.\mathrm{S}_{1} \sqcup \cdots \sqcup \mathrm{S}_{n}, \leq\right)$ via $\leq_{1,2}, \ldots, \leq_{n-1, n}$ and extending the bonds $f_{1}, \ldots, f_{n}$ to $\bar{f}: \overline{\mathrm{S}} \longrightarrow \mathbb{N}$ by $\bar{f}(x, y)=1$ if $\mathrm{S}_{i} \ni x<y \in \mathrm{S}_{i+1}$, we have that a standard monomial is just an LS path in $\mathrm{L}(\overline{\mathrm{S}}, \leq, \bar{f})$.

We assume throughout the rest of this paper that any LS monomial $\ell_{1} \cdots \ell_{r}$ is such that $\ell_{i} \in \mathrm{L}_{1}\left(\mathrm{~S}_{k_{i}}\right)$ with $k_{1} \leq \cdots \leq k_{r}$.

\section{LS algebras over multiposet}

Let $\mathrm{R}$ be a commutative ring with 1 . In this section we define an LS algebra over a multiposet $\mathrm{S}=\left(\mathrm{S}_{i}, \leq_{i}, f_{i}, \leq_{i, j}, \phi_{i, j}\right)$ as an algebra having as $\mathrm{R}$-basis the set of standard LS monomials. Further we ask that in the expressions of a monomial $\ell_{1} \cdots \ell_{r}$ in terms of $\mathrm{R}$-linear combination of standard monomials $\ell_{1, i} \cdots \ell_{r, i}$ (the straightening relations), only the standard monomials satisfying a permutation variant of the lexicographic order appear.

Let us begin defining this order. Let $N=\operatorname{lcm}\left\{f(x, y) \mid x, y \in \mathrm{S}_{i}, x<y, i=\right.$ $1, \ldots, n\}$. If $\ell=\left(a_{1}<\cdots<a_{s} ; 0=x_{0}<x_{1}<\cdots<x_{s}=1\right)$ is an LS path of degree 1 , we associate with $\ell$ a corresponding word $\omega(\ell)$ in the alphabet $\mathrm{S}_{1} \sqcup \cdots \sqcup \mathrm{S}_{n}$

$$
\omega(\ell)=a_{1}^{N\left(x_{1}-x_{0}\right)} \cdots a_{s}^{N\left(x_{s}-x_{s-1}\right)} .
$$

Notice that $N\left(x_{i}-x_{i-1}\right) \in \mathbb{N}$ by definition of $N$ and LS path. We extend the map $\omega$ to monomials by $\omega\left(\ell_{1} \cdots \ell_{r}\right)=\omega\left(\ell_{1}\right) \cdots \omega\left(\ell_{r}\right)$ (juxtapposition of words). We have len $\omega\left(\ell_{1} \cdots \ell_{r}\right)=N r$, where len $\omega$ is the length of the word $\omega$. Note also that $\omega(\ell)=\omega\left(\ell^{\prime}\right)$ for $\ell, \ell^{\prime} \in \mathrm{L}_{1}(\mathrm{~S})$ implies $\ell=\ell^{\prime}$.

As the reader can see this definition is just a simpler version of the notion of word for LS paths given in [5]. We have restated it here in this form for the sake of readability since in the application to the multicone in next section, we will need just this special type of word.

We have a natural action of the symmetric group $\mathfrak{S}_{m}$, on the set of words $\omega=\omega_{1} \cdots \omega_{m}$ of length $m$, namely: $\sigma \omega=\omega_{\sigma(1)} \cdots \omega_{\sigma(m)}$. Suppose we are given two LS monomials $\ell_{1} \cdots \ell_{r}, \ell_{1}^{\prime} \cdots \ell_{r}^{\prime}$ of the same multidegree $\mathbf{a}=\left(a_{1}, \ldots, a_{n}\right)$, then we write

$$
\ell_{1} \cdots \ell_{r} \leq \ell_{1}^{\prime} \cdots \ell_{r}^{\prime}
$$

if $\omega\left(\ell_{1} \cdots \ell_{r}\right) \leq_{\text {lex }} \sigma \omega\left(\ell_{1}^{\prime} \cdots \ell_{r}^{\prime}\right)$ for any $\sigma \in \mathfrak{S}_{N a_{1}} \times \cdots \times \mathfrak{S}_{N a_{n}} \subset \mathfrak{S}_{N r}$. In the same way we define $\ell_{1} \cdots \ell_{r} \leq_{\text {rlex }} \ell_{1}^{\prime} \cdots \ell_{r}^{\prime}$ using reverse lexicographic order.

Another notion we will use in the sequel is the following: an LS monomial $\ell_{1} \cdots \ell_{r}$ with multidegree $\left(a_{1}, \ldots, a_{n}\right)$ admits as a standard form the monomial 
$\bar{\ell}_{1} \cdots \bar{\ell}_{r}$ if $\ell^{(i)}=\ell_{a_{1}+\cdots+a_{i-1}+1} \circ \cdots \circ \ell_{a_{1}+\cdots+a_{i}} \in \mathrm{L}_{a_{i}}\left(\mathrm{~S}_{i}\right)$ and $\bar{\ell}_{1} \cdots \bar{\ell}_{r} \in \mathrm{M}_{r}^{s}$, where $\bar{\ell}_{a_{1}+\cdots+a_{i-1}+1} \circ \cdots \circ \bar{\ell}_{a_{1}+\cdots+a_{i}}$ is the canonical decomposition of $\ell^{(i)}$ for $i=1, \ldots, n$. Notice that if a monomial admits a standard form then it admits a unique standard form. This is just an extension to multiposet of the notion of canonical decomposition.

Now we are finally ready to define LS algebras over a multiposet. Let $\mathrm{R}$ be a commutative ring with 1 , A a commutative $\mathrm{R}$-algebra, $j: \mathrm{L}_{1}(\mathrm{~S}) \hookrightarrow \mathrm{A}$ an injection that we extend to monomials by $j\left(\ell_{1} \cdots \ell_{r}\right)=j\left(\ell_{1}\right) \cdots j\left(\ell_{r}\right)$ (note that the map $j$ can also be extended to $\mathrm{L}_{r}\left(\mathrm{~S}_{i}\right)$ using canonical decomposition to define $j(\ell)=j\left(\ell_{1}\right) \cdots j\left(\ell_{r}\right)$ if $\ell_{1} \circ \cdots \circ \ell_{r}$ is the canonical decomposition of $\left.\ell\right)$. In the following we consider LS monomials as elements of A via the map $j$.

Definition 2.1. We say that $\mathrm{A}$ is an LS algebra over the multiposet $\mathrm{S}=\left(\mathrm{S}_{i}\right.$, $\left.\leq_{i}, f_{i}, \leq_{i, j}, \phi_{i, j}\right)$ if the following three conditions hold:

LS1: A is a free $\mathrm{R}$-module isomorphic to $\oplus_{\mathbf{a} \in \mathbb{N}^{n}} \mathrm{RM}_{\mathbf{a}}^{s}$ and this decomposition gives a multigrading for $\mathrm{A}$,

LS2: if $\ell_{1} \cdots \ell_{r}$ is any LS monomial and

$$
\ell_{1} \cdots \ell_{r}=\sum u_{i} \ell_{1, i} \cdots \ell_{r, i}, \quad u_{i} \in \mathrm{R} \backslash 0
$$

is the straightening relation (S.R.) for $\ell_{1} \cdots \ell_{r}$ guaranteed by LS1 then for all $i$ we have

$$
\ell_{1, i} \cdots \ell_{r, i} \leq \ell_{1} \cdots \ell_{r}
$$

LS3: if $\ell_{1} \cdots \ell_{r}$ admits as standard form $\bar{\ell}_{1} \cdots \bar{\ell}_{r}$ then the monomial $\bar{\ell}_{1} \cdots \bar{\ell}_{r}$ appears in the straightening relation for $\ell_{1} \cdots \ell_{r}$ with coefficient $u \in \mathrm{R}^{\times}$, the group of invertible elements of $\mathrm{R}$.

Further, if all such $u$ equal 1 then we say that $\mathrm{A}$ is special.

The simplest straightening relations are those in the following

Definition 2.2. If $\mathrm{A}$ is an LS algebra over the multiposet $\mathrm{S}$ whose S.R. are

$$
\ell_{1} \cdots \ell_{r}= \begin{cases}u \bar{\ell}_{1} \cdots \bar{\ell}_{r} & \text { if } \ell_{1} \cdots \ell_{r} \text { admits the standard form } \bar{\ell}_{1} \cdots \bar{\ell}_{r}, u \in \mathrm{R}^{\times} \\ 0 & \text { otherwise }\end{cases}
$$

then we say that A is a discrete LS algebra.

Notice that, given a multiposet $\mathrm{S}$, there exists a unique (up to isomorphisms) discrete special LS algebra over $\mathrm{S}$. We denote this algebra by $\mathrm{A}\{\mathrm{S}\}$. In particular if all bonds equal 1 , i.e. $f_{i}(x, y)=1$ for any pair $x<y$ in $\mathrm{S}_{i}$ and any $i=1, \ldots, n$, then we denote the unique discrete special algebra by $\mathrm{R}\{\mathrm{S}\}$. This is just the Stanley-Reisner ring of the multiposet (see [21]). Clearly this algebra is isomorphic to the quotient of the ring $\mathrm{R}\left[t_{a}\right]_{a \in \mathrm{S}_{1} \sqcup \cdots \sqcup \mathrm{S}_{n}}$ by the ideal $\mathrm{I}_{\mathrm{S}}$ generated by all products $t_{a_{1}} \cdots t_{a_{m}}$ with $a_{1} \cdots a_{m} \notin \mathrm{M}^{s}$ (here we are identifing LS paths and elements of $\mathrm{S}_{1} \sqcup \cdots \sqcup \mathrm{S}_{n}$ since every LS path is of the form $\left.(a ; 0<1)\right)$. 
In analogy with [5], there is another way to look at the order requirement in the S.R.: the order on monomials defined above is equivalent to the lexicographic order with respect to any $n$-tuple of total order refinements of the given partial orders. Let us explain this in details.

Consider an $n$-tuple of total orders $\left(\leq_{1}^{t}, \ldots, \leq_{n}^{t}\right)$ with $\leq_{i}^{t}$ refining $\leq_{i}$ on $\mathrm{S}_{i}$, $i=1, \ldots, n$. Given a word $w=w_{1} w_{2} \cdots$ with $w_{1}, \ldots, w_{a_{1}} \in \mathrm{S}_{1}, w_{a_{1}+1}, \ldots$, $w_{a_{1}+a_{2}} \in \mathrm{S}_{2}$ and so on, we denote by $[w]_{\left(\leq_{1}^{t}, \ldots, \leq_{n}^{t}\right)}$ the word obtained by reordering $w_{1} \cdots w_{a_{1}}$ by $\leq_{1}^{t}, w_{a_{1}+1} \cdots w_{a_{1}+a_{2}}$ by $\leq_{2}^{t}$ and so on. We define the multidegree of $w$ as $\left(a_{1}, \ldots, a_{n}\right)$.

Now let $v=v_{1} v_{2} \cdots, w=w_{1} w_{2} \cdots$ be two words such that $v_{h}, w_{h} \in \mathrm{S}_{i_{h}}$ with $i_{1} \leq i_{2} \leq \cdots$. Notice that $v$ and $w$ have the same multidegree $\left(a_{1}, \cdots, a_{n}\right)$.

Proposition 2.1. The following are equivalent:

(1) $v \leq_{\text {lex }} \sigma w$ for any $\sigma \in \mathfrak{S}_{a_{1}} \times \cdots \times \mathfrak{S}_{a_{n}}$,

(2) $v \leq_{\text {lex }}[w]_{\left(\leq_{1}^{t}, \ldots, \leq_{n}^{t}\right)}$ for any $n$-tuple of total orders $\left(\leq_{1}^{t}, \ldots, \leq_{n}^{t}\right)$ with $\leq_{i}^{t}$ refining $\leq_{i}$ on $\mathrm{S}_{i}, i=1, \ldots, n$.

Proof.Let $v^{1}=v_{1} v_{2} \cdots v_{a_{1}}, v^{2}=v_{a_{1}+1} v_{a_{1}+2} \cdots v_{a_{1}+a_{2}}$ and so on, and let $w^{1}$, $w^{2}, \ldots$ be defined in the same way. Then (1) is equivalent to: there exist $h \geq 1$, $\sigma_{1} \in \mathfrak{S}_{a_{1}}, \ldots, \sigma_{h-1} \in \mathfrak{S}_{a_{h-1}}$ such that (a) $v^{i} \leq_{\text {lex }} \sigma w^{i}$ for any $\sigma \in \mathfrak{S}_{a_{i}}$ and any $i=1, \ldots, h$ and (b) $v^{i}=\sigma_{i} w^{i}$ for $i=1, \ldots, h-1$. Hence we can use the analogous statement for a unique poset proved in [5].

Recall that the S.R. for an LS algebra over a unique poset can be derived by the quadratic S.R. as seen in [5] (proving the equivalence of LS2 and LS2' there). Consider now an LS algebra over a multiposet. Using the same proof of [5] it is easy to show that the S.R. are generated by the quadratic S.R. and by the S.R. for weak standard monomials which are not standard. But, in general, the quadratic S.R. do not suffice, as the simple Example 4.2 below shows.

Suppose now that we have a canonical multiposet S. Since every weak standard monomial is standard, the quadratic S.R. generate all S.R. Moreover the standard monomials over $\mathrm{S}$ can be identified with LS paths in $\mathrm{L}(\overline{\mathrm{S}}, \leq, \bar{f})$ (see the last remark at the end of Section 1). However an LS algebra over $\mathrm{S}$ is not an LS algebra over $(\overline{\mathrm{S}}, \leq, \bar{f})$ since the order requirement for the S.R. over $\mathrm{S}$ is weaker than the requirement over $(\overline{\mathrm{S}}, \leq, \bar{f})$.

\section{Deformation to discrete algebras}

Now we want to develop a deformation theory for LS algebras over multiposet. This will be completely analogous to the deformation theory for LS algebras over a (unique) poset seen in [5]. Given an LS algebra A, we introduce its "indiscrete part" $\mathrm{I}(\mathrm{A})$ and prove that $\mathrm{A}$ is a discrete algebra if and only if $\mathrm{I}(\mathrm{A})$ is empty. 
Then we prove a property of the "minimal elements" of I(A) and we index I(A) by choosing minimal elements. Finally we use this indexing in order to construct a flat family over $\mathrm{R}[t]$ whose generic fibre is $\mathrm{A}$ and whose special fibre is $\mathrm{A}_{0}$, a dicrete algebra. Further if $A$ is special then $A_{0}$ is special too, i.e. $A_{0}=A\{S\}$. While in [5] the same deformation was obtained via a step by step procedure, here we avoid doing so, by using the indexing of $\mathrm{I}(\mathrm{A})$ by minimal elements.

If $\omega=\omega_{1} \omega_{2} \cdots \omega_{m}$ is a word in the alphabet $S_{1} \sqcup \cdots \sqcup S_{n}$ and $a$ is an element of $\mathrm{S}_{1} \sqcup \cdots \sqcup \mathrm{S}_{n}$, let us define $\omega(a)$ as the number of $\omega_{i}$ that are equal to $a$.

Definition 3.1. Let $\mathrm{A}$ be an LS algebra over the multiposet $\mathrm{S}$. The indiscrete part of $\mathrm{A}, \mathrm{I}(\mathrm{A})$ is the set of elements a of $\mathrm{S}_{1} \sqcup \cdots \sqcup \mathrm{S}_{n}$ such that there exists a S.R. $\ell_{1} \cdots \ell_{r}=\sum r_{i} \ell_{1, i} \cdots \ell_{r, i}$ and there exists an $i$ such that $\omega\left(\ell_{1, i} \cdots \ell_{r, i}\right)(a) \supsetneqq$ $\omega\left(\ell_{1} \cdots \ell_{r}\right)(a)$.

Proposition 3.1. The algebra $\mathrm{A}$ is discrete if and only if $\mathrm{I}(\mathrm{A})$ is empty.

Proof. Note that if $\bar{\ell}_{1} \cdots \bar{\ell}_{r}$ is the standard form of the monomial $\ell_{1} \cdots \ell_{r}$ then there exists a permutation $\sigma \in \mathfrak{S}_{N r}$ such that $\omega\left(\bar{\ell}_{1} \cdots \bar{\ell}_{r}\right)=\sigma \omega\left(\ell_{1} \cdots \ell_{r}\right)$. Now it is clear that if $\mathrm{A}$ is a discrete algebra then $\mathrm{I}(\mathrm{A})=\varnothing$. Conversely, let $\ell_{1} \cdots \ell_{r}=\sum_{i=1}^{t} u_{i} \ell_{1, i} \cdots \ell_{r, i}$ be a straightening relation with $t \geq 1$. Since $\operatorname{len} \omega\left(\ell_{1} \cdots \ell_{r}\right)=\operatorname{len} \omega\left(\ell_{1, i} \cdots \ell_{r, i}\right)$ and $\omega\left(\ell_{1} \cdots \ell_{r}\right)(a) \leq \omega\left(\ell_{1, i} \cdots \ell_{r, i}\right)(a)$ for any $a \in \mathrm{S}_{1} \cup \cdots \cup \mathrm{S}_{n}$, using $\mathrm{I}(\mathrm{A})=\varnothing$, we have $\omega\left(\ell_{1} \cdots \ell_{r}\right)(a)=\omega\left(\ell_{1, i} \cdots \ell_{r, i}\right)(a)$ for any $a$. Hence there exists a $\sigma \in \mathfrak{S}_{N r}$ such that $\omega\left(\ell_{1, i} \cdots \ell_{r, i}\right)=\sigma \omega\left(\ell_{1} \cdots \ell_{r}\right)$. Clearly this implies that $\ell_{1} \cdots \ell_{r}$ admits $\ell_{1, i} \cdots \ell_{r, i}$ as its standard form, since $\omega\left(\ell_{1, i} \cdots \ell_{r, i}\right)$ is totally ordered. Then $t=1$ since the standard form is unique. Hence $\mathrm{A}$ is discrete.

Let $H$ be a subset of $\mathrm{S}_{1} \sqcup \cdots \sqcup \mathrm{S}_{n}$. We say that $a \in H \cap \mathrm{S}_{h}$ is a minimal element of $H$ if $H \cap \mathrm{S}_{k}=\varnothing$ for any $k<h$ and $b \in H \cap \mathrm{S}_{h}$ implies $b \nless a$. Notice that a non-empty set admits minimal elements (recall our initial finiteness assumption on posets). Now let $\ell_{1} \cdots \ell_{r}=\sum u_{i} \ell_{1, i} \cdots \ell_{r, i}$ be a straightening relation in A, fix an index $i$ and suppose that $\mathrm{I}(\mathrm{A}) \cap\left(\operatorname{supp} \ell_{1, i} \cup \cdots \cup \operatorname{supp} \ell_{r, i}\right)$ is not empty. Let $a$ be a minimal element of this set. We have

Proposition 3.2. $\omega\left(\ell_{1} \cdots \ell_{r}\right)(a) \leq \omega\left(\ell_{1, i} \cdots \ell_{r, i}\right)(a)$.

Proof. Let $j$ be such that $a \in \mathrm{S}_{j}$ and let $k$ be such that $\ell_{k} \notin \mathrm{L}_{1}\left(\mathrm{~S}_{j}\right)$, and $\ell_{k+1} \in \mathrm{L}_{1}\left(\mathrm{~S}_{j}\right)$. Then the same is true for $\ell_{k, i}$ and $\ell_{k+1, i}$ since $\mathrm{A}$ is multigraded by LS1. Arguing as in the proof of Proposition 3.1 above we have that $\ell_{1, i} \cdots \ell_{k, i}$ is the standard form of $\ell_{1} \cdots \ell_{k}$. Hence we can assume $j=1$. Clearly only the LS-paths supported in $\mathrm{S}_{1}$ are relevant, so we can also suppose that any LS-path appearing in the two monomials $\ell_{1} \cdots \ell_{r}, \ell_{1, i} \cdots \ell_{r, i}$ is in $\mathrm{L}_{1}\left(\mathrm{~S}_{1}\right)$. Now we have reduced ourselves to proving the same statement for an LS algebra over a unique poset, which is done in [5]. 
Now let $\left\{a_{0}, \ldots, a_{q}\right\}=\mathrm{I}(\mathrm{A})$ be an indexing of the elements of $\mathrm{I}(\mathrm{A})$ such that $a_{i}$ is a minimal element of $\left\{a_{i}, \ldots, a_{q}\right\}$. Fix an integer $\mathrm{T} \gg 1$ and define the following map

$$
\mathrm{L}_{1}(\mathrm{~S}) \ni \ell \mapsto \tau(\ell)=\sum_{i=0}^{q} \omega(\ell)\left(a_{i}\right) \mathrm{T}^{q-i}
$$

and for a monomial $\ell_{1} \cdots \ell_{r}$ set $\tau\left(\ell_{1} \cdots \ell_{r}\right)=\tau\left(\ell_{1}\right)+\cdots+\tau\left(\ell_{r}\right)$. This map has the following "nice" property that is the key tool for our deformation theorem.

Proposition 3.3. $\tau\left(\ell_{1} \cdots \ell_{r}\right) \leq \tau\left(\ell_{1, i} \cdots \ell_{r, i}\right)$ for any S.R.

$$
\ell_{1} \cdots \ell_{r}=\sum u_{i} \ell_{1, i} \cdots \ell_{r, i}
$$

and for any $i$. Furthermore, if $\ell_{1} \cdots \ell_{r}$ admits as standard form $\bar{\ell}_{1} \cdots \bar{\ell}_{r}$ then $\tau\left(\ell_{1} \cdots \ell_{r}\right)=\tau\left(\bar{\ell}_{1} \cdots \bar{\ell}_{r}\right)$.

Proof. The inequality is a direct consequence of Proposition 3.2. For the second statement we have noted that $\omega\left(\bar{\ell}_{1} \cdots \bar{\ell}_{r}\right)$ is a permutation of $\omega\left(\ell_{1} \cdots \ell_{r}\right)$.

Now we can define our flat family over $\mathrm{R}[t]$ for a new indeterminate $t$. Consider the following multiplicative filtration by ideals of $\mathrm{A}$

$$
\mathrm{I}_{0}=\mathrm{A} \supset \mathrm{I}_{1} \supset \mathrm{I}_{2} \supset \cdots
$$

where $\mathrm{I}_{n}$ is the ideal generated by monomials $\ell_{1} \cdots \ell_{r}$ such that $\tau\left(\ell_{1} \cdots \ell_{r}\right) \geq n$. By Proposition 3.3 above we have that the ideal $\mathrm{I}_{n}$ has an $\mathrm{R}$-basis given by the set of standard LS path monomials $\ell_{1} \cdots \ell_{r}$ such that $\tau\left(\ell_{1} \cdots \ell_{r}\right) \geq n$. Then we construct the Rees algebra corresponding to this filtration

$$
\mathcal{A}=\cdots \oplus \mathrm{A} t^{2} \oplus \mathrm{A} t \oplus \mathrm{I}_{1} t^{-1} \oplus \mathrm{I}_{2} t^{-2} \oplus \cdots
$$

and, as in [5], we obtain the following theorem

Theorem 3.1. Suppose that $\mathrm{R}$ is a field. Then $\mathcal{A}$ is a flat deformation whose general fiber is $\mathrm{A}$ and whose special fiber is a discrete algebra $\mathrm{A}_{0}$. Further if $\mathrm{A}$ is a special algebra then $\mathrm{A}_{0}=\mathrm{A}\{\mathrm{S}\}$.

As a consequence of our deformation theory, we give a result on the CohenMacaulay property for canonical multiposets and special algebras. Let $\mathrm{S}=\left(\mathrm{S}_{i}\right.$, $\left.\leq_{i}, f_{i}, \leq_{i, j}, \phi_{i, j}\right)$ be a canonical multiposet and let $(\overline{\mathrm{S}}, \leq)$ be the associated poset constructed at the end of last section. Suppose that A is a special LS algebra over S. We have:

Theorem 3.2. A is Cohen-Macaulay if and only if the Stanley-Reisner ring $\mathrm{R}\{\overline{\mathrm{S}}\}$ is Cohen-Macaualy, i.e. if and only if the poset $(\overline{\mathrm{S}}, \leq)$ is Cohen-Macaulay. 
Proof. Same proof as Theorem 30 in [5].

\section{Application to the multicone}

Let $X$ be the weight lattice of a symmetrizable Kac-Moody algebra $\mathfrak{g}$ and let $X^{+}$be the set of dominant weights. Fix a dominant weight $\lambda$, let $\mathrm{V}_{\lambda}$ be the corresponding irreducible complex representation, $\mathrm{W}_{\lambda}$ be the stabilizer of $\lambda$ in the Weyl group $\mathrm{W}$ of $\mathfrak{g}$ and denote by $\leq$ the Bruhat order on $\mathrm{S}_{\lambda}=\mathrm{W} / \mathrm{W}_{\lambda}$.

Recall that the character of $\mathrm{V}_{\lambda}$ can be combinatorially described by means of path models (see [18] for details). Denote by $\Pi$ the set of all piecewise linear paths in $X \otimes_{\mathbb{Z}} \mathbb{R}$ starting at the origin and ending on an integral weight, and let $\Pi^{+}$be the subset of paths having their image inside the dominant Weyl chamber. Fix a path $\pi \in \Pi^{+}$ending in $\lambda$. The path model $\mathbb{B}_{\pi}$ of $\mathrm{V}_{\lambda}$ is the set of paths obtained from $\pi$ by applying the root operators $f_{\alpha}, e_{\alpha}$. In particular the path model of $\pi_{\lambda}: t \mapsto t \lambda$ is the set of LS paths of shape $\lambda$. Recall now that $\left(\mathrm{S}_{\lambda}, \leq\right)$ has a set of bonds given by $f_{\lambda}(\sigma, \tau)=\left(\sigma(\lambda), \beta^{\vee}\right)$ for an adjacent pair $\sigma=s_{\beta}(\tau)<\tau$ and that LS paths of degree 1 over $\left(\mathrm{S}_{\lambda}, \leq, f_{\lambda}\right)$ correspond to LS paths of shape $\lambda$ as seen in [5].

Now let $\lambda_{1}, \lambda_{2}$ be dominant weights. The posets $\left(\mathrm{S}_{\lambda_{1}}, \leq\right),\left(\mathrm{S}_{\lambda_{2}}, \leq\right)$ can be lifted to a poset $\left(\mathrm{S}_{\lambda_{1}, \lambda_{2}}, \leq\right)$ by declaring $\mathrm{S}_{\lambda_{1}} \ni \sigma_{1}<\sigma_{2} \in \mathrm{S}_{\lambda_{2}}$ whenever there exist $w_{1}, w_{2} \in \mathrm{W}$ such that $w_{1} \leq w_{2}$ in $\mathrm{W}, \omega_{1}=\sigma_{1} \bmod \mathrm{W}_{\lambda_{1}}$ and $\omega_{2}=$ $\sigma_{2} \bmod \mathrm{W}_{\lambda_{2}}$ (see Section 7). Note that the posets $\mathrm{S}_{\lambda_{1}, \lambda_{2}}$ and $\mathrm{S}_{\lambda_{2}, \lambda_{1}}$ are different.

Now consider the graph $\mathcal{G}(\pi)$ associated to a path $\pi$ whose set of vertices is $\mathbb{B}_{\pi}$, with an arrow $\eta \longrightarrow \eta^{\prime}$ coloured by a simple root $\alpha$ if $f_{\alpha}(\eta)=\eta^{\prime}$. Recall (see [18],[19]) that the map $\pi_{\lambda_{1}} * \pi_{\lambda_{2}} \mapsto \pi_{\lambda_{2}} * \pi_{\lambda_{1}}$ extends to an isomorphism of graphs $\phi_{\lambda_{1}, \lambda_{2}}: \mathcal{G}\left(\pi_{\lambda_{1}} * \pi_{\lambda_{2}}\right) \longrightarrow \mathcal{G}\left(\pi_{\lambda_{2}} * \pi_{\lambda_{1}}\right)$. Also note that $\mathbb{B}_{\pi_{\lambda_{1}} * \pi_{\lambda_{2}}}$ is exactly the set of pairs of LS paths $\left(\eta, \eta^{\prime}\right)$ such that $\max \operatorname{supp} \eta \leq \min \operatorname{supp} \eta^{\prime}$ in $\mathrm{S}_{\lambda_{1}, \lambda_{2}}$ (see Theorem10.1 [19]).

Example 4.1. Let us see a simple instance of this graph isomorphism. Take G to be the simple group $\mathrm{SL}_{3}$ and let $\omega_{1}, \omega_{2}$ be the two fundamental weights. Consider the two paths $\pi_{\omega_{1}}: t \mapsto t \omega_{1}$ and $\pi_{\omega_{2}}: t \mapsto t \omega_{2}$. The coloured graph obtained starting with the path $\pi_{\omega_{1}} * \pi_{\omega_{2}}$ (resp. $\pi_{\omega_{2}} * \pi_{\omega_{1}}$ ) is the one showed in Figure 1 (resp. Figure 2). The isomorphism can be traced after the corresponding arrows in the following two graphs.

There is, however, a general pattern here. Suppose that G is of type A. Then the isomorphism of graphs $\phi_{\omega_{i}, \omega_{j}}$, where $\omega_{i}$ and $\omega_{j}$ are fundamental weights, can be computed using the jeu de taquin and the tableau representation for paths (see [17] for generalities on jeu de taquin and in particular [4] for this computation).

Let us see a slightly more complicated example. Let us keep $\mathrm{G}=\mathrm{SL}_{3}$, and compute the isomorphism $\phi_{\omega_{1}, \lambda}$, where $\lambda=\omega_{1}+\omega_{2}$ as above. The graph corresponding to $\pi_{\omega_{1}} * \pi_{\lambda}$ and to $\pi_{\lambda} * \pi_{\omega_{1}}$ are showed in Figures 3 and 4 below. To the best of our knowledge there is no simple combinatorial technique, like tableaux 
and jeu de taquin, to fully understand this computation.

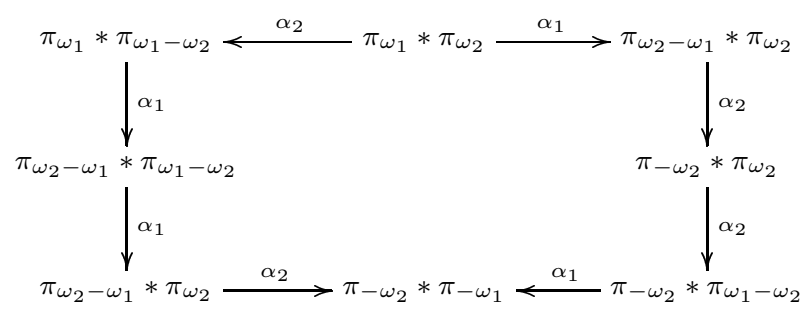

Figure 1. The graph $\mathcal{G}\left(\pi_{\omega_{1}} * \pi_{\omega_{2}}\right)$

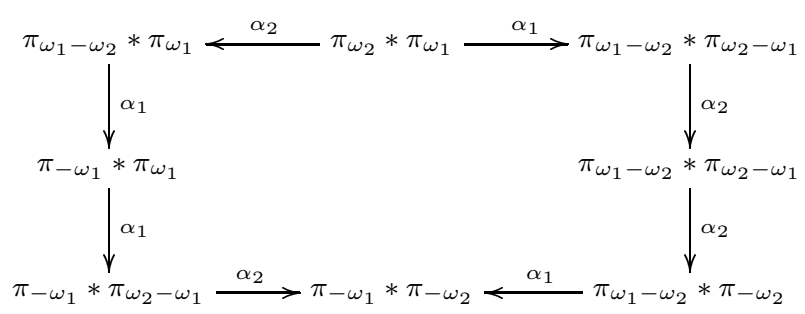

Figure 2. The graph $\mathcal{G}\left(\pi_{\omega_{2}} * \pi_{\omega_{1}}\right)$

Let us return to the general construction. Now we have all we need to define a multiposet. Let $\lambda_{1}, \lambda_{2}, \ldots, \lambda_{n}$ be dominant weights. We have the posets with bonds $\left(\mathrm{S}_{\lambda_{i}}, \leq, f_{\lambda_{i}}\right)$ and for $i \neq j$ we can define $\leq_{i, j}$ using the lifting of $\left(\mathrm{S}_{\lambda_{i}}, \leq\right)$ and $\left(\mathrm{S}_{\lambda_{j}}, \leq\right)$ to $\left(\mathrm{S}_{\lambda_{i}, \lambda_{j}}, \leq\right)$. We also have the swappings $\phi_{\lambda_{i}, \lambda_{j}}$ from $\mathrm{L}_{2}^{i, j}=\mathbb{B}_{\pi_{\lambda_{i}} * \pi_{\lambda_{j}}}$ to $\mathrm{L}_{2}^{j, i}=\mathbb{B}_{\pi_{\lambda_{j}} * \pi_{\lambda_{i}}}$, hence we can define a multiposet with bonds $\left(\mathrm{S}_{\lambda_{i}} ; f_{\lambda_{i}} ; \phi_{\lambda_{i}, \lambda_{j}}\right)$ that we denote by $\mathrm{S}\left(\lambda_{1}, \ldots, \lambda_{n}\right)$. We note here that a monomial $\pi_{1} \cdots \pi_{n}$ of LS-paths of shape $\lambda_{1}, \ldots, \lambda_{n}$ is standard (in the sense of definition in Section 1) if and only if $\pi_{1} * \cdots * \pi_{n} \in \mathcal{G}\left(\pi_{\lambda_{1}} * \cdots * \pi_{\lambda_{n}}\right)$ (see again Theorem $10.1[19])$.

Now let $\mathrm{R}=k$ be an algebraically closed field and let $\mathrm{G}$ be the simply connected semisimple group corresponding to $\mathfrak{g}$ and let $\mathrm{B} \subset \mathrm{G}$ be a Borel subgroup corresponding to the dominant weights $X^{+}$. Given an LS path $\pi$ of shape $\lambda$ we can associate with it a section $p_{\pi} \in H^{0}\left(\mathrm{G} / \mathrm{B}, \mathcal{L}_{\lambda}\right)$ of the line bundle $\mathcal{L}_{\lambda}=\mathrm{G} \times{ }_{\mathrm{B}} k_{-\lambda}$ (see [20],[16]). Consider now the algebra $\mathrm{A}\left(\lambda_{1}, \ldots, \lambda_{n}\right)=$ $\oplus_{\mathbf{a} \in \mathbb{N}^{n}} H^{0}\left(\mathrm{G} / \mathrm{B}, \mathcal{L}_{a_{1} \lambda_{1}+\cdots+a_{n} \lambda_{n}}\right)$. We have the following 


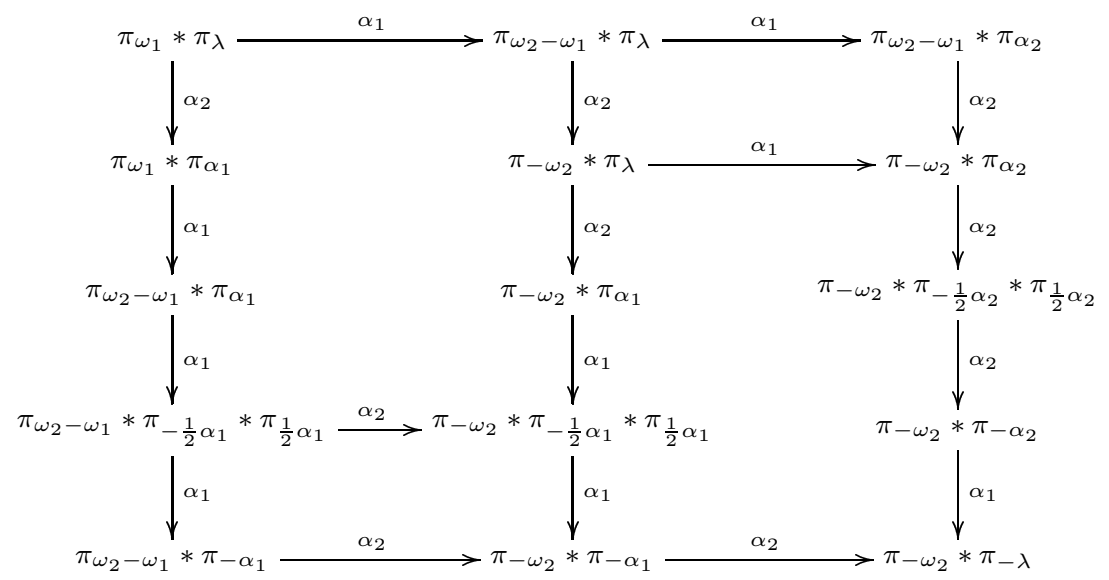

Figure 3. The graph $\mathcal{G}\left(\pi_{\omega_{1}} * \pi_{\lambda}\right)$.

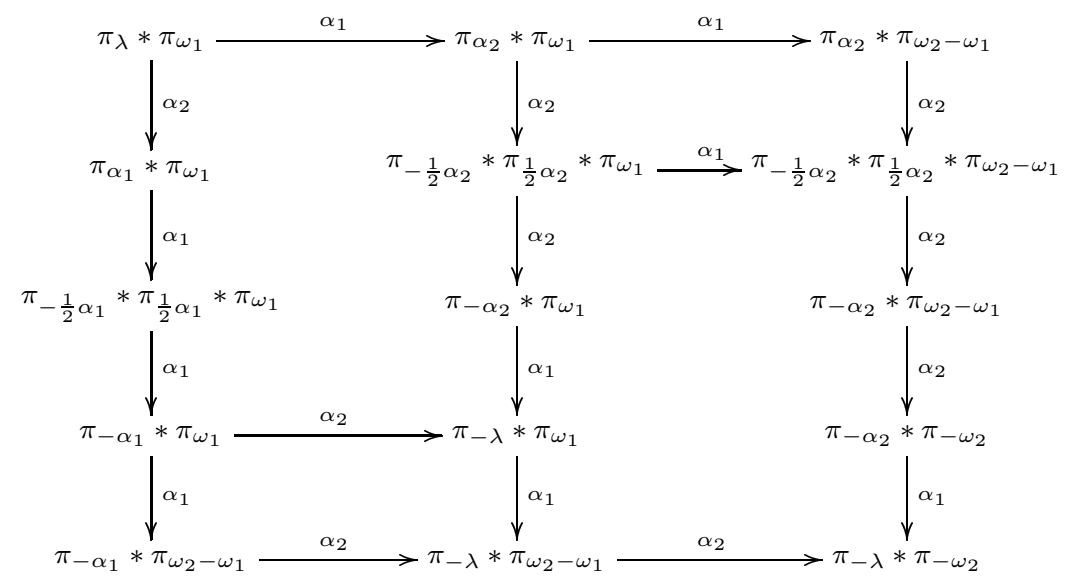

Figure 4. The graph $\mathcal{G}\left(\pi_{\lambda} * \pi_{\omega_{1}}\right)$.

Proposition 4.1. $\mathrm{A}\left(\lambda_{1}, \ldots, \lambda_{n}\right)$ is a special LS algebra over the multiposet $\mathrm{S}\left(\lambda_{1}, \ldots, \lambda_{n}\right)$. It admits a flat deformation to the special discrete algebra $\mathrm{A}\left\{\mathrm{S}\left(\lambda_{1}, \ldots, \lambda_{n}\right)\right\}$.

Proof. The second statement is a consequence of the first and of Theorem 3.1. The map $\pi \mapsto p_{\pi}$ gives an injection of $\mathrm{L}_{1}\left(\mathrm{~S}\left(\lambda_{1}, \ldots, \lambda_{n}\right)\right)$ in $\mathrm{A}\left(\lambda_{1}, \ldots, \lambda_{n}\right)$. We claim that with respect to this injection $\mathrm{A}\left(\lambda_{1}, \ldots, \lambda_{n}\right)$ verifies LS1, LS2 and LS3 for an LS algebra over a multiposet.

Let $\lambda=\underbrace{\lambda_{1}+\cdots+\lambda_{1}}_{a_{1}}+\cdots+\underbrace{\lambda_{n}+\cdots+\lambda_{n}}_{a_{n}}$, where $a_{1}, \ldots, a_{n}$ are non negative integers. Then the set of standard monomials $p_{\pi_{1}} \cdots p_{\pi_{a_{1}}} \cdots p_{\pi_{a_{1}+\cdots+a_{n}}}$ 
forms a basis of $\mathrm{V}_{\lambda}^{*} \simeq H^{0}\left(\mathrm{G} / \mathrm{B}, \mathcal{L}_{\lambda}\right) \quad([20]$, Theorem 4). This proves LS1 for $\mathrm{A}\left(\lambda_{1}, \ldots, \lambda_{n}\right)$ since any $k$-linear relation among monomials must be homogeneous in $\mathrm{A}\left(\lambda_{1}, \ldots, \lambda_{n}\right)$.

We know that the relations for $\mathrm{A}\left(\lambda_{1}, \ldots, \lambda_{n}\right)$ are generated by the relations of degree 2 (see [12]). Now, it is clear that if the quadratic relations fulfil LS2 then any relation fulfils LS2. So we can suppose $n=2$.

Proposition 7.3 of [16] can be generalized verbatim to LS paths of different shapes. Then the inequality obtained there can be read as our lexicographic requirement in LS2 as in [5]. Moreover, $\mathrm{A}\left(\lambda_{1}, \ldots, \lambda_{n}\right)$ is special using Corollary 7.4 of [16] for different shapes.

Now let $\mathcal{F}=\mathrm{G} / \mathrm{P}$ be the flag variety corresponding to the parabolic subgroup $\mathrm{P}$ and let $\omega_{1}, \ldots, \omega_{n}$ be the fundamental weights of $\mathrm{G}$ corresponding to $\mathrm{P}$. We call any intersection of a toric variety with a projective subspace a linear section of the toric variety. Then we have

Theorem 4.1. The multicone over the flag variety $\mathcal{F}$ admits a flat deformation to a union of linear sections of toric varieties.

Proof. First we note that the S.R. for $\mathrm{A}\left(\omega_{1}, \ldots, \omega_{\ell}\right)$ are defining relations for the multicone over $\mathcal{F}$. Then applying the previous proposition to $\mathrm{A}\left(\omega_{1}, \ldots, \omega_{\ell}\right)$ we obtain a discrete algebra $\mathrm{A}_{0}=\mathrm{A}\left\{\mathrm{S}\left(\omega_{1}, \ldots, \omega_{\ell}\right)\right\}$. It is clear that $\mathrm{A}_{0}$ is the coordinate ring of a union of linear sections of toric varieties, where linear sections come from the equations $\ell_{1} \cdots \ell_{r}=0$ for the weak standard, non standard monomials $\ell_{1} \cdots \ell_{r}$.

Example 4.2. In the proof of the previous Proposition 4.1 we used [12] to derive the S.R. for the algebra $\mathrm{A}\left(\lambda_{1}, \ldots, \lambda_{n}\right)$ from the quadratic S.R. Note that the property of being generatated by quadratic S.R. is no longer true in the deformed algebra $\mathrm{A}\left\{\mathrm{S}\left(\lambda_{1}, \ldots, \lambda_{n}\right)\right\}$ if weak-standard is different from standard. Also for $\mathrm{G}=\mathrm{SL}_{4}$ : consider the fundamental weights with indexing $\omega_{2}, \omega_{3}, \omega_{1}$; the tableau $(24,134,2)$ is not standard but it is weak-standard, so there is no way to derive $p_{24} p_{134} p_{2}=0$ in $\mathrm{A}\left\{\mathrm{S}\left(\omega_{2}, \omega_{3}, \omega_{1}\right)\right\}$ from the set of quadratic S.R.: $p_{i_{1} \cdots i_{s}} p_{j_{1} \cdots j_{t}}=$ 0 where $\left(i_{1} \cdots i_{s}, j_{1} \cdots j_{t}\right)$ is a non standard tableau.

\section{The good string case}

In most cases we can obtain a more precise version of Theorem 4.1. Suppose $\mathrm{G}$ is a simple Kac-Moody group, let $\mathrm{D}$ be the Dynkin diagram of $\mathrm{G}$ and let $\mathrm{B} \subset \mathrm{P}=\cap_{\alpha \in \mathrm{DP}_{\mathrm{P}}} \mathrm{P}_{\alpha}$ be a parabolic subgroup, where $\mathrm{P}_{\alpha}$ is the maximal parabolic subgroup corresponding to a simple root $\alpha$ and $\mathrm{D}_{\mathrm{P}} \subset \mathrm{D}$ is some subset. Now fix an indexing of $\mathrm{D}_{\mathrm{P}}$, say $\mathrm{D}_{\mathrm{P}}=\left\{\alpha_{1}, \ldots, \alpha_{n}\right\}$, and, for $h=1, \ldots, n-1$ let $\mathrm{D}_{i}$ be the connected component of $\mathrm{D} \backslash \alpha_{i+1}$ containing $\alpha_{i}$. 
Definition 5.1. We say that $\mathrm{P}$ has the good string property (g.s.p.) if for some indexing $\alpha_{1}, \ldots, \alpha_{n}$ of $\mathrm{D}_{\mathrm{P}}$ we have $\alpha_{j} \notin \mathrm{D}_{i}$ for all $1 \leq i<j \leq n$.

This definition of good string is equivalent to the one given in [19]. Notice that Section 1 of [19] can be easily generalized to Kac-Moody algebra using minimal representatives instead of maximal representatives for elements of Coxeter group/parabolic subgroup in Lemma 11.1, Corollary 2 and Lemma 11.3 there. Therefore we have

Lemma 5.1. [see [19], Lemma 11.3] If $\mathrm{P}$ has the g.s.p. then the multiposet $\mathrm{S}\left(\omega_{1}, \ldots, \omega_{n}\right)$ is canonical, i.e. all weak standard monomials are standard.

Theorem 5.1. If the parabolic subgroup $\mathrm{P}$ has the good-string property then the multicone over the flag variety $\mathcal{F}=\mathrm{G} / \mathrm{P}$ admits a deformation to a union of toric varieties. Moreover the multicone over $\mathcal{F}$ is Cohen-Macaulay, hence normal, whenever $\mathrm{G}$ is of finite type.

Proof. As in the proof of Theorem 4.1, A $\left(\omega_{1}, \ldots, \omega_{\ell}\right)$ admits a flat deformation to $\mathrm{A}\left\{\mathrm{S}\left(\omega_{1}, \ldots, \omega_{\ell}\right)\right\}$. Since $\mathrm{S}\left(\omega_{1}, \ldots, \omega_{\ell}\right)$ is canonical when $\mathrm{P}$ has the good string property, we can improve the result of Theorem 4.1 obtaining that $\mathcal{F}$ can be flat deformed to a union of toric varieties. Further $\mathrm{A}\left(\omega_{1}, \ldots, \omega_{\ell}\right)$ is Cohen-Macaulay if and only if $S\left(\omega_{1}, \ldots, \omega_{\ell}\right)$ is Cohen-Macauly by Theorem 3.2. But $S\left(\omega_{1}, \ldots, \omega_{\ell}\right)$ is a Bruhat poset and, if we assume that $\mathrm{G}$ is of finite type, $\mathrm{S}\left(\omega_{1}, \ldots, \omega_{\ell}\right)$ is lexicographically shellable, as we will prove in Section 9, and hence Cohen-Macaulay. Finally the normality of the multicone follows from non singularity of the flag variety.

In order to characterize parabolic subgroups with the g.s.p. we give the following simple criterion that covers finite and affine types.

Proposition 5.1. (1) Let G be a simple Kac-Moody group whose Dynkin diagram $\mathrm{D}$ has no loop. A parabolic subgroup $\mathrm{P}=\cap_{\alpha \in \mathrm{D}_{\mathrm{P}}} \mathrm{P}_{\alpha}$ has the g.s.p. if and only if there exists a subset $\mathcal{P}$ of $\mathrm{D}$ containing $\mathrm{D}_{\mathrm{P}}$ and isomorphic, as a graph, to a (connected) segment.

(2) Let $\mathrm{G}$ be of type $\mathrm{A}_{\ell}^{(1)}$. A parabolic subgroup $\mathrm{P}=\mathrm{P}_{\alpha_{1}} \cap \cdots \cap \mathrm{P}_{\alpha_{n}}$ has the g.s.p. if and only if $n \leq 2$.

Proof. Let $\mathcal{P}$ be a segment containing $\mathrm{D}_{\mathrm{P}}$, say $\mathcal{P}=\left\{\alpha_{1}, \ldots, \alpha_{m}\right\}$. We choose the indexing of $\mathrm{D}_{\mathrm{P}}$ as a subset of $\mathcal{P}$, say $\mathrm{D}_{\mathrm{P}}=\left\{\alpha_{h_{1}}, \ldots, \alpha_{h_{n}}\right\}$ for some $1 \leq$ $h_{1}<\cdots<h_{n} \leq m$. We claim that $\mathrm{D}_{\mathrm{P}}$ has the g.s.p. with respect to this indexing. Indeed if $\alpha_{h_{j}} \in \mathrm{D}_{i}$ for some $j>i$ then $\mathrm{D}$ must contain two different segments joining $\alpha_{h_{i}}$ and $\alpha_{h_{j}}$. But then $\mathrm{D}$ should contain a loop. This also 
proves (2). Now suppose that $\mathrm{P}$ has the g.s.p. and let $\mathrm{D}_{\mathrm{P}}=\left\{\alpha_{1}, \ldots, \alpha_{n}\right\}$ be an indexing such that $\alpha_{j} \notin \mathrm{D}_{i}$ for all $1 \leq i<j \leq n$. We use induction on $n$. If $n=2$ then the claim is clear since for every pair of vertices of a connected graph there exists a segment containing them. So we can suppose $n>2$. Note that $\left\{\alpha_{1}, \ldots, \alpha_{n-1}\right\}$ is an indexing of $\mathrm{D}_{\mathrm{P}} \backslash \alpha_{n}$ with the g.s.p. and hence we can suppose that there exists a segment $\mathcal{P}^{\prime}$ containing $\left\{\alpha_{1}, \ldots, \alpha_{n-1}\right\}$. Now let $\mathcal{P}^{\prime \prime}=\left\{\beta_{0}=\alpha_{n-1}, \beta_{1}, \ldots, \beta_{s}=\alpha_{n}\right\}$ be a segment joining $\alpha_{n-1}$ and $\alpha_{n}$. Let $\beta_{i} \in \mathcal{P}^{\prime} \cap \mathcal{P}^{\prime \prime}$. If $i>0$ then $\left\{\beta_{i}, \beta_{i+1}, \ldots, \beta_{s}\right\} \subset \mathrm{D}_{n-1}$ and this is impossible since $D_{\mathrm{P}}$ has the g.s.p. So $\mathcal{P}^{\prime} \cap \mathcal{P}^{\prime \prime}=\left\{\alpha_{n-1}\right\}$ and hence $\mathcal{P}=\mathcal{P}^{\prime} \cup \mathcal{P}^{\prime \prime}$ is a segment containing $\left\{\alpha_{1}, \ldots, \alpha_{n}\right\}$.

Example 5.1. If $\mathrm{G}$ is of type $\mathrm{A}_{\ell}, \mathrm{B}_{\ell}, \mathrm{C}_{\ell}, \mathrm{F}_{4}, \mathrm{G}_{2}, \mathrm{~A}_{1}^{(1)}, \mathrm{C}_{\ell}^{(1)}, \mathrm{G}_{2}^{(1)}, \mathrm{F}_{4}^{(1)}$, $\mathrm{A}_{2}^{(2)}, \mathrm{A}_{2 \ell}^{(2)}, \mathrm{D}_{\ell+1}^{(2)}, \mathrm{E}_{6}^{(2)}, \mathrm{D}_{4}^{(3)}$ (see [11]) then any parabolic subgroup has the g.s.p. In particular, the full flag variety $\mathrm{G} / \mathrm{B}$ admits a deformation to a union of toric varieties.

Using the last remark of Section 2 we have a new proof of the following result (see [12]) in the g.s.p. case:

Proposition 5.2. If $\lambda_{1}, \ldots, \lambda_{n}$ are dominant weights such that the positive lattice $\left\langle\lambda_{1}, \ldots, \lambda_{n}\right\rangle_{\mathbb{N}}$ is stabilized by some parabolic subgroups $\mathrm{P}$ of $\mathrm{G}$ having the g.s.p. then $\mathrm{A}\left(\lambda_{1}, \ldots, \lambda_{n}\right)$ is isomorphic to a polynomial ring modulo an ideal generated by quadratic relations.

\section{Coxeter groups and Bruhat order}

In the following four sections we turn to pure combinatorial methods of Coxeter groups. In this section we briefly recall the fundamental properties of Bruhat order on Coxeter groups and parabolic subgroups. Everything is well-known: proofs and further details can be found, for example, in [3], [6], [9], [8], [24] and [25].

Definition 6.1. A Coxeter group is a pair $(\mathrm{W}, \mathrm{S})$ such that $\mathrm{W}$ is a group and $\mathrm{S}$ is a distinguished set of generators of $\mathrm{W}$ such that

(1) $s^{2}=e$, for all $s \in \mathrm{S}$,

(2) $\left(s_{i} s_{j}\right)^{\left(p_{i j}\right)}=e, p_{i j} \geq 2$, for all $s_{i} \neq s_{j}$ in $\mathrm{S}$ such that $s_{i} s_{j}$ is of finite order,

is a presentation of $\mathrm{W}$.

In the sequel $(\mathrm{W}, \mathrm{S})$ is a fixed Coxeter group. If $w=s_{1} s_{2} \cdots s_{q} \in \mathrm{W}, s_{i} \in \mathrm{S}$ is an expression of $w$ in terms of the generators, we call the word $s_{1} s_{2} \cdots s_{q}$ in the alphabet $\mathrm{S}$ an expression for $w$. The length len $(w)$ of $w \in \mathrm{W}$ is the least integer $q$ for which an expression $w=s_{1} s_{2} \cdots s_{q}$ exists. Such an expression 
$w=s_{1} s_{2} \cdots s_{q}$ of minimal length is called reduced.

One of the main tools for performing computations with reduced expressions is the following property, called the exchange condition.

Theorem 6.1. Let $w=s_{1} \cdots s_{r}$ with $s_{i} \in \mathrm{S}$, not necessarily a reduced expression. Suppose $s \in \mathrm{S}$ satisfies $\operatorname{len}(w s)<\operatorname{len}(w)$. Then there exist an index $i$ for which $w s=s_{1} \cdots \hat{s}_{i} \cdots s_{r}$ (omitting $s_{i}$ ). If the expression for $w$ is reduced, then $i$ is unique.

Now we recall the definition of Bruhat order on a Coxeter group (W, S) . This is the most useful way to order a Coxeter group compatibly with the length function. Let $\mathrm{T}$ be the set of conjugates of $\mathrm{S}$ in $\mathrm{W}$, i.e., $\mathrm{T}=\left\{w s w^{-1} \mid w \in \mathrm{W}, s \in \mathrm{S}\right\}$, the elements of $\mathrm{T}$ are usually called reflections.

Definition 6.2. For two elements $w, w^{\prime}$ of $\mathrm{W}$ write $w^{\prime} \leftarrow w$ if $w^{\prime}=w t$ for some $t \in \mathrm{T}$ with $\operatorname{len}\left(w^{\prime}\right)<\operatorname{len}(w)$. Then define $w^{\prime}<w$ if there is a sequence $w^{\prime}=w_{0} \leftarrow w_{1} \leftarrow \cdots \leftarrow w_{m}=w$. The resulting relation $w^{\prime} \leq w$ gives a partial ordering of $\mathrm{W}$ having $e$ as the unique minimal element, and is called the Bruhat order.

A first remark on this definition. When $w^{\prime} \leftarrow w$ the length difference is not specified and it can be more than 1 . However, what is true is that $\leq$-adjacent elements differ in length by exactly 1 . Notice that the definition has a one-sided appearance, since we have written the $t \in \mathrm{T}$ on the right in the arrow definition. But is not hard to show that the left sided version is equivalent to the one given above. The following important characterization is very useful. Furthermore it explains the left-right symmetry of the definition.

Lemma 6.1. Let $w=s_{1} s_{2} \cdots s_{q}$ be a fixed reduced expression for $w$. Then $w^{\prime} \leq w$ if and only if there exists a reduced expression $w^{\prime}=s_{i_{1}} s_{i_{2}} \cdots s_{i_{k}}$ with $1 \leq i_{1}<i_{2}<\cdots<i_{k} \leq q$. In other words $w^{\prime}$ can be written as a sub expression of any reduced expression for $w$.

Let $\mathrm{J} \subset \mathrm{S}$ and let $\mathrm{W}_{\mathrm{J}}$ be the subgroup generated by $\mathrm{J}$ in $\mathrm{W}$. Subgroups of the form $\mathrm{W}_{\mathrm{J}}$ are called parabolic. This kind of subgroups of $\mathrm{W}$ are, in general, far from being normal. There is however a good way to represent cosets modulo $\mathrm{W}_{\mathrm{J}}$.

Lemma 6.2. Let $\mathrm{J} \subset \mathrm{S}$ and let $\mathrm{W}_{\mathrm{J}}$ be the corresponding parabolic subgroup. Then the set

$$
\mathrm{W}^{\mathrm{J}}=\{w \in \mathrm{W} \mid w s>w \text { for all } s \in \mathrm{J}\}
$$

satisfies:

(1) each element $w \in \mathrm{W}$ can be factored in a unique way as $w=u v$ with 
$u \in \mathrm{W}^{\mathrm{J}}$ and $v \in \mathrm{W}_{\mathrm{J}}$,

(2) if $w=u v$ as in (1) then $\operatorname{len}(w)=\operatorname{len}(u)+\operatorname{len}(v)$,

(3) each element $u \in \mathrm{W}^{\mathrm{J}}$ is the unique minimal element of the coset $u \mathrm{~W}_{\mathrm{J}}$ and in particular is the unique element of minimal length of such coset.

We call $\mathrm{W}^{\mathrm{J}}$ the set of minimal representatives of $\mathrm{W}$ modulo $\mathrm{W}_{\mathrm{J}}$. In view of this lemma, it is not surprising that the partial order on $\mathrm{W}^{\mathrm{J}} \cong \mathrm{W} / \mathrm{W}_{\mathrm{J}}$ induced by the Bruhat order on $\mathrm{W}$ is significant. In what follows, we shall denote the induced order on $\mathrm{W}^{\mathrm{J}}$ also by $\leq$.

Notice that a parabolic subgroup $\mathrm{W}_{\mathrm{I}}$ is itself the Coxeter group $\left(\mathrm{W}_{\mathrm{I}}, \mathrm{I}\right)$. We have defined the Bruhat order on $\mathrm{W}_{\mathrm{I}}$ as a restriction of the Bruhat order of $\mathrm{W}$. We can also consider the Bruhat order of $W_{I}$ as the Coxeter group $\left(W_{I}, I\right)$. We have

Lemma 6.3. If $\mathrm{I} \subset \mathrm{S}$, the Bruhat order of the Coxeter group $\left(\mathrm{W}_{\mathrm{I}}, \mathrm{I}\right)$ agrees with the restriction on $\mathrm{W}_{\mathrm{I}}$ of the Bruhat order of $\mathrm{W}$.

Now we collect some results, to be used in the sequel, whose proofs are standard.

Lemma 6.4. Let $u \in \mathrm{W}^{\mathrm{J}}, v \in \mathrm{W}_{\mathrm{J}}$. If $u=s_{1} \cdots s_{p}, v=s_{1}^{\prime} \cdots s_{q}^{\prime}$ are reduced expressions for $u$ and $v$ then $s_{1} \cdots s_{p} s_{1}^{\prime} \cdots s_{q}^{\prime}$ is a reduced expression for $u v$.

Lemma 6.5. Assume that $u \in \mathrm{W}^{\mathrm{J}}, w \in \mathrm{W}, w>u$ and $\operatorname{len}(w)=\operatorname{len}(u)+1$. Then either $w \in \mathrm{W}^{\mathrm{J}}$ or $w=u s$ for some $s \in \mathrm{J}$.

Lemma 6.6. Let $w \leq w^{\prime}$ in $\mathrm{W}$ and $s \in \mathrm{S}$. Then either $w s \leq w^{\prime}$ or else $w s \leq w^{\prime} s$, or both.

As a final remark notice that if the Coxeter group $(W, S)$ is finite then there exists a unique longest element $w_{0}$ in $\mathrm{W}$ that is also a unique maximal element for the Bruhat order on W. Furthermore if $\mathrm{W}$ is finite, the parabolic subgroup $\mathrm{W}_{\mathrm{I}}, \mathrm{I} \subset \mathrm{S}$, is finite and hence it admits a longest element $w_{0, \mathrm{I}}$. By Lemma 6.3 we can consider $w_{0, \mathrm{I}}$ either as the longest element of the Coxeter group $\left(\mathrm{W}_{\mathrm{I}}, \mathrm{I}\right)$ or as the unique maximal element of $W_{\mathrm{I}}$ as subset of $\mathrm{W}$.

We end this section by setting some notation. If $w$ is an element of $\mathrm{W}$, then we denote by $[w]^{\mathrm{I}}$ the minimal representative of $w$ modulo the parabolic subgroup $\mathrm{W}_{\mathrm{I}}$. Further, we denote by $w_{0}^{\mathrm{I}}$ the longest element of $\mathrm{W}^{\mathrm{I}}$ in case $\mathrm{W}$ is finite.

\section{Bruhat posets}

In this section we introduce the main objects of our interest and we prove some related properties. Let $(\mathrm{I}, \mathrm{J})$ be a pair of subsets of $\mathrm{S}$, and consider the associated 
sets of minimal representatives $\mathrm{W}^{\mathrm{I}}, \mathrm{W}^{\mathrm{J}}$. These are posets, i.e. partially ordered sets with respect to the Bruhat order as seen in Section 6. Now consider the disjoint union $\mathrm{W}^{(\mathrm{I}, \mathrm{J})}=\mathrm{W}^{\mathrm{I}} \sqcup \mathrm{W}^{\mathrm{J}}$. We want to define an order on the set obtained by "glueing" the two posets $\mathrm{W}^{\mathrm{I}}, \mathrm{W}^{\mathrm{J}}$. For details see [13],[14], [15] and [7].

Definition 7.1. Let $\mathrm{I}_{1}=\mathrm{I}, \mathrm{I}_{2}=\mathrm{J}$ and let $\tau, \sigma \in \mathrm{W}^{(\mathrm{I}, \mathrm{J})}$. Set $\tau \preceq \sigma$ if

(1) $\tau \in \mathrm{W}^{\mathrm{I}_{h}}, \sigma \in \mathrm{W}^{\mathrm{I}_{k}}$ with $h \leq k$ and

(2) there exist $\tau^{\prime}, \sigma^{\prime} \in \mathrm{W}$ such that $\tau^{\prime} \leq \sigma^{\prime}$ and $\tau^{\prime}=\tau \bmod \mathrm{W}_{\mathrm{I}_{h}}, \sigma^{\prime}=$ $\sigma \bmod \mathrm{W}_{\mathrm{I}_{k}}$.

The following simple lemma shows that the definition really extends the Bruhat order on $\mathrm{W}^{\mathrm{I}}$ and on $\mathrm{W}^{\mathrm{J}}$.

Lemma 7.1. Let $\tau, \sigma \in \mathrm{W}^{\mathrm{I}}$. There exist $\tau^{\prime}, \sigma^{\prime} \in \mathrm{W}$ such that $\tau^{\prime} \leq \sigma^{\prime}$ and $\tau^{\prime}=\tau \bmod \mathrm{W}_{\mathrm{I}}, \sigma^{\prime}=\sigma \bmod \mathrm{W}_{\mathrm{I}}$ if and only if $\tau \leq \sigma$.

Proof. Let $u, v \in \mathrm{W}_{\mathrm{I}}$ be such that $\tau^{\prime}=\tau u, \sigma^{\prime}=\sigma v$. First of all notice that $\tau \leq \tau u=\tau^{\prime} \leq \sigma^{\prime}$ by Lemma 6.4 and so we can suppose $u=e$, i.e. $\tau^{\prime}=\tau$. Now let $\sigma=s_{1} s_{2} \cdots s_{r}$ and $v=s_{1}^{\prime} s_{2}^{\prime} \cdots s_{t}^{\prime}$ be reduced expressions for $\sigma$ and $v$. From $\tau \leq \sigma v$ we have $\tau=s_{i_{1}} s_{i_{2}} \cdots s_{i_{p}} s_{j_{1}}^{\prime} s_{i_{2}}^{\prime} \cdots s_{j_{q}}^{\prime}$ for some $1 \leq i_{1}<i_{2}<\cdots<i_{p} \leq r$, $1 \leq j_{1}<j_{2}<\cdots j_{q} \leq t$. But then $\tau=s_{i_{1}} s_{i_{2}} \cdots s_{i_{p}} \bmod \mathrm{W}_{\mathrm{I}}$ and since $\tau \in \mathrm{W}^{\mathrm{I}}$ we have $\tau=s_{i_{1}} s_{i_{2}} \cdots s_{i_{p}} \leq \sigma$.

The relation $\preceq$ is a partial order, as seen easily from Lemma 7.1 to show antisymmetry. We call the poset $\left(\mathrm{W}^{(\mathrm{I}, \mathrm{J})}, \preceq\right)$ the glueing of $\mathrm{W}^{\mathrm{I}}$ and $\mathrm{W}^{\mathrm{J}}$. Notice that, by definition, although $\mathrm{W}^{(\mathrm{I}, J)}$ and $\mathrm{W}^{(\mathrm{J}, \mathrm{I})}$ agree as sets, they differ as partially ordered sets. In view of last lemma we will freely write $\tau \preceq \sigma$ or $\tau \leq \sigma$ if $\tau, \sigma \in \mathrm{W}^{\mathrm{I}}$ or $\tau, \sigma \in \mathrm{W}^{\mathrm{J}}$. Further, note that using Lemma 7.1 it is easy to prove that (2) in Definition 7.1 is equivalent to:

(2') there exist $\bar{\tau}, \bar{\sigma} \in \mathrm{W}^{\mathrm{I}_{1} \cap \mathrm{I}_{2}}$ such that $\bar{\tau} \leq \bar{\sigma}$ and $\bar{\tau}=\tau \bmod \mathrm{W}_{\mathrm{I}_{h}}, \bar{\sigma}=$ $\sigma \bmod \mathrm{W}_{\mathrm{I}_{k}}$.

Indeed, clearly $\left(2^{\prime}\right) \Rightarrow(2)$ since $\mathrm{W}^{\mathrm{I}_{1} \cap \mathrm{I}_{2}} \subset \mathrm{W}$. Conversely, let $\bar{\tau}=\left[\tau^{\prime}\right]^{\mathrm{I}_{1} \cap \mathrm{I}_{2}}, \bar{\sigma}=$ $\left[\sigma^{\prime}\right]^{\mathrm{I}_{1} \cap \mathrm{I}_{2}}$. Then $\bar{\tau} \leq \bar{\sigma}$ by Lemma 7.1 and $[\bar{\tau}]^{\mathrm{I}_{1}}=\left[[\tau]^{\mathrm{I}_{1} \cap \mathrm{I}_{2}}\right]^{\mathrm{I}_{1}}=\tau, \quad[\bar{\sigma}]^{\mathrm{I}_{2}}=$ $\left[\left[\sigma^{\prime}\right]^{\mathrm{I}_{1} \cap \mathrm{I}_{2}}\right]^{\mathrm{I}_{2}}=\sigma$.

Example 7.1. Let $\mathrm{W}=\mathfrak{S}_{3}$, the group of permutations on three letters. It is a Coxeter group with respect to generators $\mathrm{S}=\left\{s_{1}=(12), s_{2}=(23)\right\}$. Let $\mathrm{I}=\left\{s_{1}\right\}, \mathrm{J}=\left\{s_{2}\right\}$. Then the posets $\mathrm{W}^{(\mathrm{I}, \mathrm{J})}, \mathrm{W}^{(\mathrm{J}, \mathrm{I})}$ are the ones depicted in Figure 5 .

Now we want to study some aspects of the adjacency relation in $\mathrm{W}^{(\mathrm{I}, \mathrm{J})}$ in the case of a finite W. So we assume through the rest of this paper that $\mathrm{W}$ is a finite Coxeter group unless otherwise specified. The next part of 


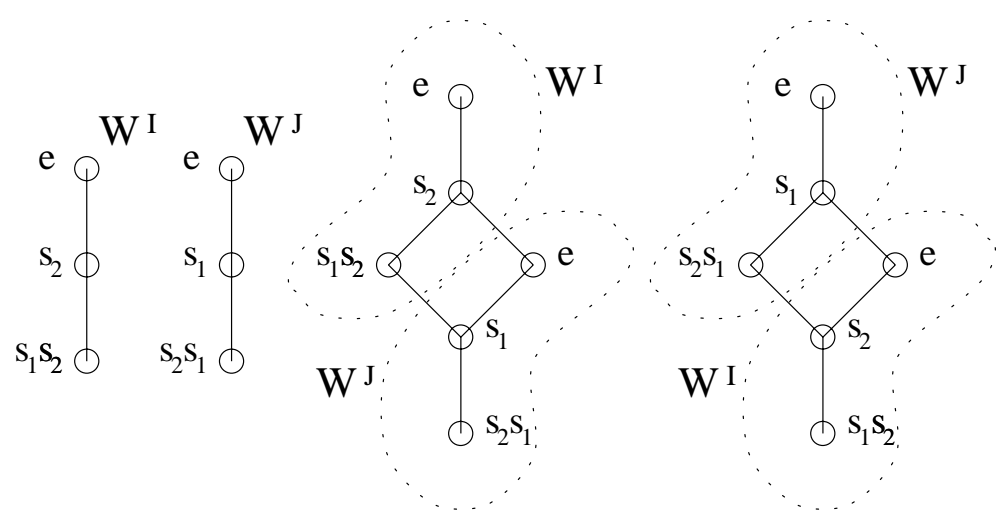

Figure 5. The posets $\mathrm{W}^{\mathrm{I}}, \mathrm{W}^{\mathrm{J}}, \mathrm{W}^{(\mathrm{I}, \mathrm{J})}, \mathrm{W}^{(\mathrm{J}, \mathrm{I})}$.

this section is devoted to the proof of a certain "homogeneity" in the adjacency relation between $\mathrm{W}^{\mathrm{I}} \hookrightarrow \mathrm{W}^{(\mathrm{I}, \mathrm{J})}$ and $\mathrm{W}^{\mathrm{J}} \hookrightarrow \mathrm{W}^{(\mathrm{I}, \mathrm{J})}$.

Definition 7.2. We write $\tau \triangleleft \sigma$ if $\tau \in \mathrm{W}^{\mathrm{I}}, \sigma \in \mathrm{W}^{\mathrm{J}}, \tau \preceq \sigma$ and they are adjacent, i.e. there does not exist $\tau^{\prime} \in \mathrm{W}^{\mathrm{I}}$ such that $\tau \preceq \tau^{\prime} \preceq \sigma$ and there does not exist $\sigma^{\prime} \in \mathrm{W}^{\mathrm{J}}$ such that $\tau \preceq \sigma^{\prime} \preceq \sigma$.

Let us introduce some sets related to the adjacency relation $\triangleleft$.

$$
\begin{aligned}
& \mathrm{W}_{+}^{\mathrm{I}}=\left\{\tau \in \mathrm{W}^{\mathrm{I}} \mid \text { there exists } \sigma \in \mathrm{W}^{\mathrm{J}} \text {, such that } \tau \triangleleft \sigma\right\} \\
& \mathrm{W}_{-}^{\mathrm{J}}=\left\{\sigma \in \mathrm{W}^{\mathrm{J}} \mid \text { there exists } \tau \in \mathrm{W}^{\mathrm{I}} \text {, such that } \tau \triangleleft \sigma\right\}
\end{aligned}
$$

Further, throughout this section $w$ will be the element $w=\left[w_{0, J}\right]^{\mathrm{I}}$, i.e. the minimal representative of $w_{0, \mathrm{~J}}$, the longest element of $\mathrm{W}_{\mathrm{J}}$, modulo the parabolic subgroup $W_{I}$. We fix once and for all reduced expressions

$$
\begin{aligned}
w_{0, \mathrm{~J}} & =s_{1} s_{2} \cdots s_{r} \\
w & =s_{i_{1}} s_{i_{2}} \cdots s_{i_{t}}
\end{aligned}
$$

where $1 \leq i_{1}<i_{2}<\cdots<i_{t} \leq r$. Notice that $w \in \mathrm{W}^{\mathrm{I}} \cap \mathrm{W}_{\mathrm{J}}$.

The following lemma is a key result for our purpose.

Lemma 7.2. If $\sigma \in \mathrm{W}_{-}^{\mathrm{J}}$ then $\sigma w \in \mathrm{W}^{\mathrm{I}}$.

Proof. Suppose that $\sigma w$ does not belong to $\mathrm{W}^{\mathrm{I}}$. Then there exists $s \in \mathrm{I}$ such that $\sigma w s<\sigma w$. This yields $\sigma^{\prime} \doteq[\sigma w s]^{\mathrm{J}} \leq \sigma$, using Lemma 7.1 and the fact that $w \in \mathrm{W}_{\mathrm{J}}$. But we have

$$
\begin{aligned}
& \sigma w s=[\sigma w]^{\mathrm{I}} \bmod \mathrm{W}^{\mathrm{I}}, \\
& \sigma w s=\sigma^{\prime} \bmod \mathrm{W}^{\mathrm{J}},
\end{aligned}
$$


which implies $[\sigma w]^{\mathrm{I}} \preceq \sigma^{\prime} \preceq \sigma$. Hence $\sigma^{\prime}=\sigma$, since $\sigma \in \mathrm{W}_{-}^{\mathrm{J}}$.

Now fix a reduced expression $\sigma=s_{1}^{\prime} \cdots s_{p}^{\prime}$. Then a reduced expression for $\sigma w s$ can be obtained by omitting a simple reflection in

$$
\sigma w=s_{1}^{\prime} \cdots s_{p}^{\prime} s_{i_{1}} s_{i_{2}} \cdots s_{i_{t}}
$$

by the exchange condition in Theorem 6.1. Now we consider the two possible cases. If $\sigma w s=s_{1}^{\prime} \cdots s_{p}^{\prime} s_{i_{1}} \cdots \hat{s}_{i_{k}} \cdots s_{i_{t}}$, for some $1 \leq k \leq t$ we deduce $w s=s_{i_{1}} \cdots \hat{s}_{i_{k}} \cdots s_{i_{t}}$ and this is impossible, since $w \in \mathrm{W}^{\mathrm{I}}, s \in \mathrm{W}_{\mathrm{I}}$. In the other case $\sigma w s=s_{1}^{\prime} \cdots \hat{s}_{i_{k}}^{\prime} \cdots s_{p}^{\prime} w$, for some $1 \leq k \leq p$, and since $s \in \mathrm{W}_{\mathrm{J}}$, we find $\sigma=\sigma^{\prime}=[\sigma w s]^{\mathrm{J}}=\left[s_{1}^{\prime} \cdots \hat{s}_{i_{k}}^{\prime} \cdots s_{p}^{\prime}\right]^{\mathrm{J}}$ and this is impossible too as $\sigma \in \mathrm{W}^{\mathrm{J}}$.

The next step is the proof of the following.

Lemma 7.3. If $\mathrm{W}^{\mathrm{I}} \ni \tau \triangleleft \sigma \in \mathrm{W}^{\mathrm{J}}$ then $\tau=\sigma w$.

Proof. We know that $\sigma w \in \mathrm{W}^{\mathrm{I}}$ by previous lemma. Let us show that $\sigma w \preceq \sigma$. Indeed let $\epsilon \doteq \sigma w_{0, \mathrm{~J}}$, then we have

$$
\begin{aligned}
\epsilon & =\sigma \bmod \mathrm{W}_{\mathrm{J}} \\
\epsilon & =\sigma w \bmod \mathrm{W}_{\mathrm{I}}
\end{aligned}
$$

and so $\sigma w \leq \sigma$.

Suppose now that $\tau^{\prime} \in \mathrm{W}^{\mathrm{I}}$ is such that $\tau^{\prime} \leq \sigma$. Then there exist $\bar{\tau}, \bar{\sigma} \in \mathrm{W}$ such that

$$
\begin{aligned}
\bar{\tau} & =\tau^{\prime} \bmod \mathrm{W}_{\mathrm{I}} \\
\bar{\sigma} & =\sigma \bmod \mathrm{W}_{\mathrm{J}}, \\
\bar{\tau} & \leq \bar{\sigma} \text { in } \mathrm{W} .
\end{aligned}
$$

We have $\bar{\sigma}=\sigma v$ for some $v \in \mathrm{W}_{\mathrm{J}}$, hence $\sigma v \leq \sigma w_{0, \mathrm{~J}}$. So $\bar{\tau} \leq \sigma w_{0, \mathrm{~J}}$ and using Lemma 7.1 we find $\tau^{\prime} \leq \sigma w$.

Let us denote by $\rho_{w}: \mathrm{W} \rightarrow \mathrm{W}$ the map given by right multiplication by $w$, i.e. the map $\mathrm{W} \ni v \mapsto v w \in \mathrm{W}$. Obviously $\rho_{w}$ is a bijection, $\mathrm{W}$ being a group. In the following theorem we see that this map describes the new adjacency relation in $\mathrm{W}^{(\mathrm{I}, \mathrm{J})}$ obtained by glueing.

Theorem 7.1. The map $\rho_{w}: \mathrm{W}_{-}^{\mathrm{J}} \rightarrow \mathrm{W}_{+}^{\mathrm{I}}$ is a poset isomorphism. Moreover $\mathrm{W}_{+}^{\mathrm{I}}=\left(\mathrm{W}^{\mathrm{J}} w\right) \cap \mathrm{W}^{\mathrm{I}}$ and $\mathrm{W}_{-}^{\mathrm{J}}=\mathrm{W}^{\overline{\mathrm{J}}} \cap\left(\mathrm{W}^{\mathrm{I}} w^{-1}\right)$.

Proof. Let $\sigma \in \mathrm{W}_{-}^{\mathrm{J}}$. Then there exists $\tau \in \mathrm{W}_{+}^{\mathrm{I}}$ such that $\tau \triangleleft \sigma$, and by theorem above $\tau=\sigma w=\rho_{w}(\sigma)$. Hence the map $\rho_{w}$ is well defined. On the other hand if $\tau \in \mathrm{W}_{+}^{\mathrm{I}}$ then there exists $\sigma \in \mathrm{W}^{\mathrm{J}}$ such that $\tau \triangleleft \sigma$. Hence $\sigma \in \mathrm{W}_{-}^{\mathrm{J}}$ and $\tau=\sigma w$, so $\rho_{w}$ is surjective. $\rho_{w}$ is also clearly injective.

Now let $\sigma_{1} \preceq \sigma_{2}$ in $\mathrm{W}_{-}^{\mathrm{J}}$, i.e. $\sigma_{1} \leq \sigma_{2}$. Fix a reduced expression $\sigma_{2}=$ $s_{1}^{\prime} \cdots s_{p}^{\prime}$, then we have $\sigma_{1}=s_{j_{1}}^{\prime} \cdots s_{j_{q}}^{\prime}$ for some indexes $1 \leq j_{1}<j_{2}<\cdots<j_{q} \leq$ 
$p$ by Lemma 6.1. Then $\sigma_{2} w_{0, \mathrm{~J}}=s_{1}^{\prime} \cdots s_{p}^{\prime} s_{1} \cdots s_{r}$ and $\sigma_{1} w_{0, \mathrm{~J}}=s_{j_{1}}^{\prime} \cdots s_{j_{q}}^{\prime} s_{1} \cdots s_{r}$ are reduced expressions, hence $\sigma_{1} w_{0, \mathrm{~J}} \leq \sigma_{2} w_{0, \mathrm{~J}}$ in $\mathrm{W}$. This last inequality shows $\sigma_{1} w \leq \sigma_{2} w$ in $\mathrm{W}^{\mathrm{I}}$ using Lemma 7.1 , since $\left[\sigma_{1} w_{0, \mathrm{~J}}\right]^{\mathrm{I}}=\sigma_{1} w$ and $\left[\sigma_{2} w_{0, \mathrm{~J}}\right]^{\mathrm{I}}=\sigma_{1} w$.

On the other hand, suppose $\sigma_{1} w \preceq \sigma_{2} w$ in $\mathrm{W}^{\mathrm{I}}$, i.e. $\sigma_{1} w \leq \sigma_{2} w$, and choose reduced expressions $\sigma_{1}=s_{1}^{\prime \prime} \cdots s_{q}^{\prime \prime}, \sigma_{2}=s_{1}^{\prime} \cdots s_{p}^{\prime}$. Recall that $w \in \mathrm{W}_{\mathrm{J}}$, hence $\sigma_{1} w=s_{1}^{\prime \prime} \cdots s_{q}^{\prime \prime} s_{i_{1}} s_{i_{2}} \cdots s_{i_{t}}$ and $\sigma_{2} w=s_{1}^{\prime} \cdots s_{p}^{\prime} s_{i_{1}} s_{i_{2}} \cdots s_{i_{t}}$ are reduced expressions. So $\sigma_{1}=s_{1}^{\prime \prime} \cdots s_{q}^{\prime \prime}$ is a subword of $s_{1}^{\prime} \cdots s_{p}^{\prime}=\sigma_{2}$.

As for the last statement of the theorem, notice that $\mathrm{W}_{+}^{\mathrm{I}} \subset \mathrm{W}^{\mathrm{J}} w \cap \mathrm{W}^{\mathrm{I}}$ by Lemma 7.3. Now let $\tau \in \mathrm{W}^{\mathrm{J}} w \cap \mathrm{W}^{\mathrm{I}}$, i.e. $\tau=\sigma w \in \mathrm{W}^{\mathrm{I}}$ with $\sigma \in \mathrm{W}^{\mathrm{J}}$. We have $\tau \preceq \sigma$. Suppose $\tau^{\prime} \in \mathrm{W}_{+}^{\mathrm{I}}, \sigma^{\prime} \in \mathrm{W}_{-}^{\mathrm{J}}$ are such that $\tau \leq \tau^{\prime} \triangleleft \sigma^{\prime} \leq \sigma$. Then $\tau^{\prime}=\sigma^{\prime} w$ and we have $\tau=\sigma w \leq \sigma^{\prime} w=\tau^{\prime}$. But $\sigma, \sigma^{\prime} \in \mathrm{W}^{\mathrm{J}}$ and $w \in \mathrm{W}_{\mathrm{J}}$, hence as above, $\sigma \leq \sigma^{\prime}$. So $\sigma^{\prime}=\sigma, \bar{\tau}^{\prime}=\tau \in \mathrm{W}_{+}^{\mathrm{I}}$. Now the assertion for $\mathrm{W}_{-}^{\mathrm{J}}$ follows from the first part of the theorem.

Now we give a characterization of $\mathrm{W}_{+}^{\mathrm{I}}$ that will be useful in Section 9 .

Lemma 7.4. $\mathrm{W}_{+}^{\mathrm{I}}$ is the set of all elements $\tau$ of $\mathrm{W}^{\mathrm{I}}$ which admit a reduced expression of the form $\tau=s_{1}^{\prime} s_{2}^{\prime} \cdots s_{p}^{\prime} s_{i_{1}} s_{i_{2}} \cdots s_{i_{t}}$, for some $s_{1}^{\prime}, \ldots, s_{p}^{\prime} \in \mathrm{S}$

Proof. Clearly every element of $\mathrm{W}_{+}^{\mathrm{I}}$ admits such an expression by Theorem 7.1. Conversely, use induction on $p$. If $p=0$ then $\tau=w \in \mathrm{W}_{+}^{\mathrm{I}}$. Suppose $p>0$. Let $\tau^{\prime}=s_{2}^{\prime} \cdots s_{p}^{\prime} s_{i_{1}} s_{i_{2}} \cdots s_{i_{t}}$.

First of all we show $\tau^{\prime} \in \mathrm{W}^{\mathrm{I}}$. Let $s \in \mathrm{I}$. From $\tau \in \mathrm{W}^{\mathrm{I}}$ we have that $\tau s=$ $s_{1}^{\prime} \cdots s_{p}^{\prime} s$ is a reduced expression, hence $\tau^{\prime} s=s_{2}^{\prime} \cdots s_{p}^{\prime} s$ is a reduced expression and so $\tau^{\prime}=s_{2}^{\prime} \cdots s_{p}^{\prime}<s_{2}^{\prime} \cdots s_{p}^{\prime} s=\tau^{\prime} s$. This proves $\tau^{\prime} \in \mathrm{W}^{\mathrm{I}}$.

Further $s_{2}^{\prime} \cdots s_{p}^{\prime} s_{i_{1}} s_{i_{2}} \cdots s_{i_{t}}$ is a reduced expression and so, by induction $\tau^{\prime} \in$ $\mathrm{W}_{+}^{\mathrm{I}}$, i.e. $\sigma^{\prime}=s_{2}^{\prime} \cdots s_{p}^{\prime} \in \mathrm{W}_{-}^{\mathrm{J}}$. Let $\sigma=s_{1}^{\prime} \cdots s_{p}^{\prime}$. Notice that this is a reduced expression, hence $\sigma^{\prime}<\sigma$. Now we can use Lemma 6.5 to conclude either $\sigma \in \mathrm{W}^{\mathrm{J}}$ or $\sigma=\sigma^{\prime} s$, for some $s \in \mathrm{J}$.

Notice that if $\sigma \in \mathrm{W}^{\mathrm{J}}$ then $\tau=\sigma w \in \mathrm{W}_{+}^{\mathrm{I}}$ by Theorem 7.1, so we can suppose $\sigma=\sigma^{\prime} s, s \in \mathrm{J}$. From this we have $[\tau]^{\mathrm{J}}=[\sigma]^{\mathrm{J}}=\sigma^{\prime}$ and hence $\mathrm{W}^{\mathrm{I}} \ni \tau \preceq \sigma^{\prime} \in \mathrm{W}^{\mathrm{J}}$. But this is impossible since $\tau^{\prime} \triangleleft \sigma^{\prime}$ and $\tau^{\prime}<\tau<\sigma^{\prime}$.

Our next aim is to prove that the poset obtained by glueing minimal representatives are graded posets. For this we start with some preliminary lemmas.

Lemma 7.5. Let $\sigma \in \mathrm{W}_{-}^{\mathrm{J}}$. Then there exists $\sigma^{\prime} \in \mathrm{W}_{-}^{\mathrm{J}}$ such that $\sigma^{\prime} \leq \sigma$ are adjacent in $\mathrm{W}^{\mathrm{J}}$.

Proof. Let $\sigma=s_{1}^{\prime} s_{2}^{\prime} \cdots s_{p}^{\prime}$ be a reduced expression for $\sigma$ and define $\sigma^{\prime} \doteq s_{2}^{\prime} \cdots s_{p}^{\prime}$. We want to prove that this $\sigma^{\prime}$ has the desired property. 
As in the proof of the previous lemma we have $\sigma^{\prime} \in \mathrm{W}^{\mathrm{J}}$. Now we want to show $\sigma^{\prime} \in \mathrm{W}_{-}^{\mathrm{J}}$. From $\sigma \in \mathrm{W}_{-}^{\mathrm{J}}$ we have $\sigma w \in \mathrm{W}^{\mathrm{I}}$. Note that $\sigma w=$ $s_{1}^{\prime} \cdots s_{p}^{\prime} s_{i_{1}} s_{i_{2}} \cdots s_{i_{t}}$ is a reduced expression since $\sigma \in \mathrm{W}^{\mathrm{J}}, w \in \mathrm{W}_{\mathrm{J}}$ (recall $w=$ $\left.s_{i_{1}} s_{i_{2}} \cdots s_{i_{t}}\right)$. Let $s \in \mathrm{I}$. From $\sigma w \in \mathrm{W}^{\mathrm{I}}$ we have $\sigma w s>\sigma w$. Note that $\sigma w s=$ $s_{1}^{\prime} \cdots s_{p}^{\prime} s_{i_{1}} s_{i_{2}} \cdots s_{i_{t}} s$ is a reduced expression. Hence also $s_{2}^{\prime} \cdots s_{p}^{\prime} s_{i_{1}} s_{i_{2}} \cdots s_{i_{t}} s$ is a reduced expression and $s_{2}^{\prime} \cdots s_{p}^{\prime} s_{i_{1}} s_{i_{2}} \cdots s_{i_{t}} s>s_{2}^{\prime} \cdots s_{p}^{\prime} s_{i_{1}} s_{i_{2}} \cdots s_{i_{t}}$. This proves $\sigma^{\prime} w \in \mathrm{W}^{\mathrm{I}}$ and so $\sigma^{\prime} \in \mathrm{W}_{-}^{\mathrm{J}}$ by Theorem 7.1. Now the lemma is clear since $\sigma^{\prime}<\sigma$ and they are adjacent in $\mathrm{W}$.

From Lemma 7.5 we derive that $\mathrm{W}_{+}^{\mathrm{I}}$ and $\mathrm{W}_{-}^{\mathrm{J}}$ contain "enough" saturated chains.

Corollary 7.1. Given $\sigma \in \mathrm{W}_{-}^{\mathrm{J}}$ there exists a chain

$$
\mathbf{c}: \sigma=\sigma_{0}>\sigma_{1}>\cdots>\sigma_{p}=e
$$

in $\mathrm{W}_{-}^{\mathrm{J}}$ that is saturated in $\mathrm{W}^{\mathrm{J}}$. Furthermore

$$
\mathbf{c}^{\prime} \doteq \rho_{w}(\mathbf{c}): \sigma w=\sigma_{0} w>\sigma_{1} w>\cdots>\sigma_{p} w=w
$$

is a chain in $\mathrm{W}_{+}^{\mathrm{I}}$ saturated in $\mathrm{W}^{\mathrm{I}}$.

Proof. The existence of $\mathbf{c}$ follows by Lemma 7.5. Clearly $\mathbf{c}^{\prime}$ is contained in $\mathrm{W}_{+}^{\mathrm{I}}$ and is saturated by construction of $\mathbf{c}$.

Now we introduce a more general kind of object. Let $\Omega=\left(\mathrm{I}_{1}, \cdots, \mathrm{I}_{n}\right)$ be a $n$-tuple of subsets of $\mathrm{S}$. We can glue together the various posets $\left(\mathrm{W}_{\mathrm{I}_{k}}, \leq\right)$, where $\leq$ is the Bruhat order, in a unique poset $\mathrm{W}^{\Omega} \doteq \mathrm{W}^{\mathrm{I}_{1}} \sqcup \cdots \sqcup \mathrm{W}^{\mathrm{I}_{n}}$. The construction is a step by step extension of the case of two subsets I, J seen above:

let $\tau \in \mathrm{W}^{\mathrm{I}_{h}}, \sigma \in \mathrm{W}^{\mathrm{I}_{k}}$ with $h \leq k$, then define $\sigma \preceq \tau$ if and only if either $h=k$ and $\tau \leq \sigma$ or there exists a chain $\tau=\epsilon_{h} \preceq \epsilon_{h+1} \preceq \cdots \preceq \epsilon_{k}=\sigma$ such that $\epsilon_{i} \in \mathrm{W}^{\mathrm{I}_{i}}$ for $i=h, \ldots, k$ and $\epsilon_{i} \preceq \epsilon_{i+1}$ in the poset $\mathrm{W}^{\left(\mathrm{I}_{i}, \mathrm{I}_{i+1}\right)}$. It is clear that this is a partial order on $\mathrm{W}^{\Omega}$.

Remark 7.1. This definition is not equivalent to the more global following one: let $\tau \in \mathrm{W}^{\mathrm{I}_{h}}, \sigma \in \mathrm{W}^{\mathrm{I}_{k}}$ with $h \leq k$ then one defines $\tau \preceq \sigma$ if and only if there exist $\tau^{\prime}, \sigma^{\prime} \in \mathrm{W}$ such that $\tau^{\prime} \leq \sigma^{\prime}$ in $\mathrm{W}$ and $\left[\tau^{\prime}\right]^{\mathrm{I}_{h}}=\tau,\left[\sigma^{\prime}\right]^{\mathrm{I}_{k}}=\sigma$. Indeed this is not a partial order: as an example consider $\mathrm{W}=\mathrm{S}_{4}, \mathrm{I}_{1}=\left\{s_{1}, s_{3}\right\}, \mathrm{I}_{2}=$ $\left\{s_{2}, s_{3}\right\}, \mathrm{I}_{3}=\left\{s_{1}, s_{2}\right\}$ and $\sigma=s_{2} s_{1} s_{3} s_{2} \in \mathrm{W}^{\mathrm{I}_{1}}, \tau=s_{2} s_{1} \in \mathrm{W}^{\mathrm{I}_{2}}, \epsilon=e \in \mathrm{W}^{\mathrm{I}_{3}}$. Then $\sigma \leq \tau \leq \epsilon$ for both definitions, whereas $\sigma \not \leq \epsilon$ for the second one.

We call such a glueing of minimal representatives modulo parabolic subgroups of some Coxeter group, a Bruhat poset.

We recall that a finite poset $(\mathrm{P}, \leq)$ is said to be graded if it is bounded i.e. it admits a unique maximal element and a unique minimal element - and all maximal chains in $\mathrm{P}$ have the same length, where the length len(c) of a chain 
c is the number of elements in $\mathbf{c}$ minus one. This common value for the length of chains is called the dimension (or the length) of the poset. It is well-known that the posets $\left(\mathrm{W}_{\mathrm{I}}, \leq\right)$ are graded. In general we can now state the following theorem.

Theorem 7.2. $\left(\mathrm{W}^{\Omega}, \preceq\right)$ is a graded poset.

Proof. For the sake of simplicity we see the proof only for the case $\Omega=(\mathrm{I}, \mathrm{J})$, the general case presenting just a more confusing notation but no real difficulty.

Clearly $e \in \mathrm{W}^{\mathrm{I}} \subset \mathrm{W}^{(\mathrm{I}, \mathrm{J})}$ is the unique minimal element of $\mathrm{W}^{(\mathrm{I}, \mathrm{J})}$ and $w_{0}^{\mathrm{J}} \in$ $\mathrm{W}^{\mathrm{J}} \subset \mathrm{W}^{(\mathrm{I}, \mathrm{J})}$ is the unique maximal element.

Take a maximal chain $\mathbf{c}$ from $w_{0}^{\mathrm{J}}$ to $e$. Then there exist $\tau \in \mathrm{W}_{+}^{\mathrm{I}}, \sigma \in \mathrm{W}_{-}^{\mathrm{J}}$ in $\mathbf{c}$ with $\tau \triangleleft \sigma$. Break $\mathbf{c}$ up in two subchains: $\mathbf{c}_{1}$ from $\tau$ to $e$ and $\mathbf{c}_{2}$ from $w_{0}^{\mathrm{J}}$ to $\sigma$. By Corollary 7.1 there exists a chain from $\sigma$ to $e \in \mathrm{W}^{\mathrm{J}}$ contained in $\mathrm{W}_{-}^{\mathrm{J}}$ and saturated in $\mathrm{W}^{\mathrm{J}}$, say $\mathbf{c}_{2}^{\prime}$. Moreover $\mathbf{c}_{1}^{\prime} \doteq \rho_{w}\left(\mathbf{c}_{2}^{\prime}\right)$ is a chain from $\tau$ to $w$ in $\mathrm{W}_{+}^{\mathrm{I}}$ and saturated in $\mathrm{W}^{\mathrm{I}}$. Finally choose saturated chains $\mathbf{c}_{1}^{\prime \prime}$ from $w$ to $e$ in $\mathrm{W}^{\mathrm{I}}$ and $\mathbf{c}_{2}^{\prime \prime}$ from $w_{0}^{\mathrm{J}}$ to $e$ in $\mathrm{W}^{\mathrm{J}}$ (see Figure 6). Then we have

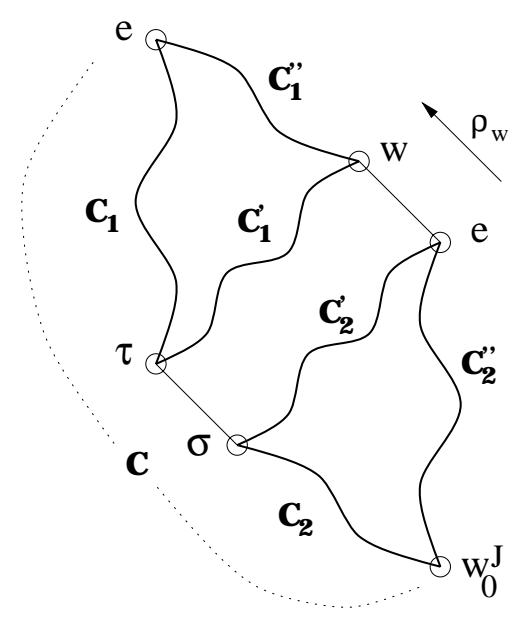

Figure 6. The various chains of the proof.

$$
\begin{aligned}
\operatorname{len}(\mathbf{c}) & =\operatorname{len}\left(\mathbf{c}_{1}\right)+1+\operatorname{len}\left(\mathbf{c}_{2}\right) \\
& =\operatorname{len}\left(\mathbf{c}_{1}^{\prime \prime}\right)+\operatorname{len}\left(\mathbf{c}_{1}^{\prime}\right)+1+\operatorname{len}\left(\mathbf{c}_{2}^{\prime \prime}\right)-\operatorname{len}\left(\mathbf{c}_{2}^{\prime}\right) \\
& =\operatorname{len}\left(\mathbf{c}_{1}^{\prime \prime}\right)+1+\operatorname{len}\left(\mathbf{c}_{2}^{\prime \prime}\right)
\end{aligned}
$$

where we have used len $\left(\mathbf{c}_{1}^{\prime}\right)=\operatorname{len}\left(\mathbf{c}_{2}^{\prime}\right)$ by construction, $\operatorname{len}\left(\mathbf{c}_{1}\right)=\operatorname{len}\left(\mathbf{c}_{1}^{\prime}\right)+\operatorname{len}\left(\mathbf{c}_{1}^{\prime \prime}\right)$ since $\mathrm{W}^{\mathrm{I}}$ is graded and len $\left(\mathbf{c}_{2}^{\prime \prime}\right)=\operatorname{len}\left(\mathbf{c}_{2}^{\prime}\right)+\operatorname{len}\left(\mathbf{c}_{2}\right)$ since $\mathrm{W}^{\mathrm{J}}$ is graded. Hence the length of $\mathbf{c}$ is independent of $\mathbf{c}$. 
Given $\tau, \sigma \in \mathrm{W}^{\Omega}$, we denote by $[\tau, \sigma]$ the closed interval $[\tau, \sigma]=\{\epsilon \in$ $\left.\mathrm{W}^{\Omega} \mid \tau \preceq \epsilon \preceq \sigma\right\}$ in $\mathrm{W}^{\Omega}$. This is a poset with order $\preceq$ induced from $\mathrm{W}^{\Omega}$. It is clear that

Corollary 7.2. Let $\tau, \sigma \in \mathrm{W}^{\Omega}$. Then $[\tau, \sigma]$ is a graded poset.

\section{Lexicographic shellable posets}

In this section we introduce the notion of lexicographic shellability for a poset. Definitions are taken from [1].

Let $(\mathrm{P}, \leq)$ be a finite graded poset with unique maximal element $1 \in \mathrm{P}$ and unique minimal element $0 \in \mathrm{P}$ of dimension $r$. Denote by $\mathcal{C}(\mathrm{P})$ the set of maximal chains

$$
\mathbf{c}: 1=x_{0}>x_{1}>\cdots>x_{r}=0
$$

from 1 to 0 in $\mathrm{P}$. We say that a map

$$
\begin{aligned}
\lambda: \mathcal{C}(\mathrm{P}) & \rightarrow \mathbb{N}^{r} \\
\mathbf{c} & \mapsto \lambda(\mathbf{c})=\left(\lambda_{1}(\mathbf{c}), \ldots, \lambda_{r}(\mathbf{c})\right)
\end{aligned}
$$

is a labeling of maximal chains of $\mathrm{P}$. We think of the integer $\lambda_{i}(\mathbf{c})$ as being associated with the edge (adjacency relation) $x_{i-1}>x_{i}$ in $\mathbf{c}$ : we are labeling $\mathbf{c}$ edgewise from top to bottom with integers.

Example 8.1. Figure 7 shows a simple example of a labeling for the two maximal chains in the poset $\mathrm{W}^{(\mathrm{I}, \mathrm{J})}$, where $\mathrm{W}=\mathrm{S}_{3}, \mathrm{~S}=\left\{s_{1}, s_{2}\right\}, \mathrm{I}=\left\{s_{1}\right\}$ and $\mathrm{J}=\left\{s_{2}\right\}$.
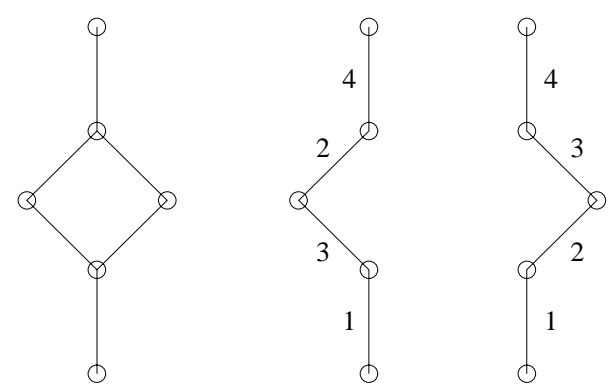

Figure 7. A labeling of $\mathrm{W}^{(\mathrm{I}, \mathrm{J})}$ as in Example 8.1.

Our first requirement for a labeling is the following.

Definition 8.1. (L1) If two maximal chains $\mathbf{c}, \mathbf{c}^{\prime}$ in $\mathrm{P}$ coincide along their first $d$ edges, $1 \leq d \leq r$, then $\lambda_{i}(\mathbf{c})=\lambda_{i}\left(\mathbf{c}^{\prime}\right)$ for $i=1,2, \ldots, d$. 
By a rooted interval $([x, y], \mathbf{c})$ we mean a pair where $[x, y]$ is an interval in $\mathrm{P}$ and $\mathbf{c}: 1=x_{0}>\cdots>x_{p}=y$ is a saturated chain from 1 to $y$ in $\mathrm{P}$. Notice that if $\lambda$ is a labeling satisfying $(\mathrm{L} 1)$ and $([x, y], \mathbf{c})$ is a rooted interval, then all maximal chains of $[x, y]$ receive an induced labeling as follows. Let $\mathbf{d}: y=z_{0}>z_{1}>\cdots>z_{h}=x$ be a maximal chain in $[x, y]$, choose an arbitrary saturated chain $\mathbf{e}$ from $x$ to 0 and consider the maximal chain $\mathbf{c}^{\prime} \doteq \mathbf{c} * \mathbf{d} * \mathbf{e} \in \mathcal{C}(\mathrm{P})$, where $*$ means composition of chains. Let $\lambda\left(\mathbf{c}^{\prime}\right)=\left(\lambda_{1}\left(\mathbf{c}^{\prime}\right), \ldots, \lambda_{r}\left(\mathbf{c}^{\prime}\right)\right)$ and define $\lambda^{\prime}(\mathbf{d})=\left(\lambda_{p+1}\left(\mathbf{c}^{\prime}\right), \lambda_{p+2}\left(\mathbf{c}^{\prime}\right), \ldots, \lambda_{p+h}\left(\mathbf{c}^{\prime}\right)\right)$. By abuse of notation we denote this by $\lambda(\mathbf{d})$ dropping the "prime". Notice that the induced labeling still satisfies (L1).

Now we focus on labelings that verify the following property.

Definition 8.2. Let $\lambda: \mathcal{C} \rightarrow \mathbb{N}^{r}$ be a labeling of the maximal chains of $\mathrm{P}$ which obeys (L1). Then we say that $\lambda$ is an L-labeling, and that $(\mathrm{P}, \leq)$ is lexicographically shellable, if $\lambda$ verifies

(L2): For every rooted interval $([x, y], \mathbf{c})$ in $\mathrm{P}$ there is a unique maximal chain $\mathbf{d}_{0}$ in $[x, y]$ whose label is increasing: $\lambda_{1}\left(\mathbf{d}_{0}\right)<\lambda_{2}\left(\mathbf{d}_{0}\right)<\cdots<\lambda_{h}\left(\mathbf{d}_{0}\right)$. Furter if $\mathbf{d}$ is any other maximal chain in $[x, y]$ then $\lambda\left(\mathbf{d}_{0}\right)<_{\operatorname{lex}} \lambda(\mathbf{d})$, where $<_{\operatorname{lex}}$ is the lexicographic order on $\mathbb{N}^{r}$.

The importance of this definition comes from the following theorem (see [23], [1] and [2]).

Theorem 8.1. If $(\mathrm{P}, \leq)$ is lexicographically shellable then $\Delta_{\mathrm{P}}$, the complex of chains of $(\mathrm{P}, \leq)$, is shellable and thus Cohen-Macaulay.

\section{Lexicographic labeling}

Now we describe a labeling of maximal chains in a closed interval of a Bruhat poset. This labeling turns out to be an L-labeling. The procedure described below is a generalization of the one provided by Björner and Wachs in [2] for minimal representatives modulo a parabolic subgroup. Indeed our labeling reduces to theirs in the case of a single parabolic subgroup.

Fix a finite Coxeter group $(\mathrm{W}, \mathrm{S})$, subsets $\mathrm{I}_{1}, \ldots, \mathrm{I}_{n}$ of $\mathrm{S}$ and consider the Bruhat poset $\mathrm{W}^{\Omega}$ for $\Omega=\left(\mathrm{I}_{1}, \ldots, \mathrm{I}_{n}\right)$ as defined above. Let $[\tau, \sigma]$ be a closed interval in $\mathrm{W}^{\Omega}$. First of all notice that no generality is lost if we suppose $\tau \in$ $\mathrm{I}_{1}, \sigma \in \mathrm{I}_{n}$. Now let $w_{2}=\left[w_{0, \mathrm{I}_{2}}\right]^{\mathrm{I}_{1}}, \ldots, w_{n}=\left[w_{0, \mathrm{I}_{n}}\right]^{\mathrm{I}_{n-1}}$ where $w_{0, \mathrm{I}_{h}}$ is the longest element of the parabolic subgroup $\mathrm{W}_{\mathrm{I}_{h}}, h=1, \ldots, n$. Choose reduced 
expressions

$$
\begin{aligned}
\sigma & =s_{1} \cdots s_{r_{1}} \\
w_{n} & =s_{r_{1}+2} \cdots s_{r_{2}} \\
& \vdots \\
w_{2} & =s_{r_{n-1}+2} \cdots s_{r_{n}}
\end{aligned}
$$

Now take a maximal chain in $[\tau, \sigma]$

$$
\mathbf{c}: \sigma=x_{0}>x_{1}>\cdots>x_{t_{1}} \triangleright x_{t_{1}+1}>\cdots>x_{t_{2}} \triangleright x_{t_{2}+1}>\cdots>x_{t}=\tau
$$

where we have stressed the $\triangleleft$-adjacency relations, i.e. we are supposing

$$
\begin{aligned}
x_{0}, \ldots, x_{t_{1}-1} & \in \mathrm{W}^{\mathrm{I}_{n}}, \\
x_{t_{1}} & \in \mathrm{W}_{-}^{\mathrm{I}_{n}} \subset \mathrm{W}^{\mathrm{I}_{n}}, \\
x_{t_{1}+1} & \in \mathrm{W}_{+}^{\mathrm{I}_{n-1}} \subset \mathrm{W}^{\mathrm{I}_{n-1}}, \\
x_{t_{1}+2}, \ldots, x_{t_{2}-1} & \in \mathrm{W}^{\mathrm{I}_{n-1}}, \\
x_{t_{2}} & \in \mathrm{W}_{-}^{\mathrm{I}_{n-1}} \subset \mathrm{W}^{\mathrm{I}_{n-1}}
\end{aligned}
$$

and so on. Consider the word $\alpha=s_{1} \cdots s_{r_{1}} \square s_{r_{1}+2} \cdots s_{r_{2}} \square \cdots \square s_{r_{n-1}+2} \cdots s_{r_{n}}$ in the alphabet $S \cup\{\square\}$, where $\square$ is a new symbol. Now, using the exchange condition, we know that there exists a uniquely determined $i_{1}$ such that $x_{1}=$ $s_{1} \cdots \hat{s}_{i_{1}} \cdots s_{r_{1}}$. Repeating this we find uniquely determined indices $i_{1}, \ldots, i_{t_{1}}$ such that

$$
x_{t_{1}}=s_{j_{1}} s_{j_{2}} \cdots s_{j_{r_{1}-t_{1}}}
$$

where $\left\{j_{1}<\cdots<j_{r_{1}-t_{1}}\right\}=\left\{1, \ldots, r_{1}\right\} \backslash\left\{i_{1}, \ldots, i_{t_{1}}\right\}$. So far, we have defined the integers $i_{1}, \ldots, i_{t_{1}}$ that correspond both to the generators removed from $s_{1} \cdots s_{r_{1}}$ and to the positions deleted in the word $\alpha$.

Now we use Theorem 7.1 to deduce $x_{t_{1}+1}=x_{t_{1}} w_{n}$. We set $i_{t_{1}+1}=r_{1}+1$, corresponding to deleting the box in position $r_{1}+1$ in the word $\alpha$, and we start the same process with the reduced expression

$$
x_{t_{1}+1}=s_{j_{1}} \cdots s_{j_{r_{1}-t_{1}}} s_{r_{1}+2} \cdots s_{r_{2}} .
$$

So we can go on till $x_{t_{2}-1}$. Then again we set $i_{t_{2}+1}=r_{2}+1$ deleting the box in position $r_{2}+1 \ldots$ Finally we reach $\tau$ having obtained the label $\lambda(\mathbf{c})=$ $\left(i_{1}, \ldots, i_{t}\right)$.

In short we keep track of removed generators in $\alpha$, considering the boxes as generators when we move from the poset $\mathrm{W}^{\mathrm{I}_{h}}$ to the poset $\mathrm{W}^{\mathrm{I}_{h-1}}, h=n, \ldots, 2$.

Let us see an example of this process.

Example 9.1. Let $\mathrm{W}^{\Omega}=\mathrm{W}^{(\mathrm{I}, \mathrm{J})}$ be as in the previous examples and consider the whole $\mathrm{W}^{\Omega}=\left[e, w_{0, \mathrm{~J}}\right]$. Start with the word $\alpha=s_{2} s_{1} \square s_{2}$. Now take the maximal chain

$$
\mathbf{c}_{1}: s_{2} s_{1}>s_{1}>e \triangleright s_{2}>e
$$


Then the label is $\lambda\left(\mathbf{c}_{1}\right)=(1,2,3,4)$ and the deletion process produces the following subwords and corresponding elements of $\mathrm{W}^{(\mathrm{I}, \mathrm{J})}$

$$
\begin{aligned}
& s_{2} \quad s_{1} \quad \square \quad s_{2} \quad \mapsto s_{2} s_{1} \in \mathrm{W}^{\mathrm{J}}, \\
& s_{1} \square s_{2} \mapsto s_{1} \in \mathrm{W}^{\mathrm{J}}, \\
& s_{2} \mapsto e \in \mathrm{W}^{\mathrm{J}}, \\
& s_{2} \mapsto s_{2} \in \mathrm{W}^{\mathrm{I}} \text {, } \\
& \varnothing \mapsto e \in \mathrm{W}^{\mathrm{I}} \text {. }
\end{aligned}
$$

Now take the other maximal chain

$$
\mathbf{c}_{2}: s_{2} s_{1}>s_{1} \triangleright s_{1} s_{2}>s_{2}>e .
$$

Then the label is $\lambda\left(\mathbf{c}_{2}\right)=(1,3,2,4)$ and corresponds to

$$
\begin{aligned}
& s_{2} \quad s_{1} \square s_{2} \mapsto s_{2} s_{1} \in \mathrm{W}^{\mathrm{J}}, \\
& s_{1} \square s_{2} \mapsto s_{1} \in \mathrm{W}^{\mathrm{J}}, \\
& s_{1} \quad s_{2} \mapsto s_{1} s_{2} \in \mathrm{W}^{\mathrm{I}}, \\
& s_{2} \mapsto s_{2} \in \mathrm{W}^{\mathrm{I}} \text {, } \\
& \varnothing \mapsto e \in \mathrm{W}^{\mathrm{I}} \text {. }
\end{aligned}
$$

Notice that our labeling assigns a unique label to an edge $\mathrm{W}^{\mathrm{I}_{h}} \ni \alpha \triangleleft \beta \in \mathrm{W}^{\mathrm{I}_{h-1}}$ : the position of the $(n-h+1)$-th boxes in $\alpha$, that does not depend on the chain considered. It follows that, in the minuscule case (see [22] for definition), the Bruhat posets are still lattices and they are shellable with respect to some edgelabeling, i.e. a labeling of maximal chains induced by a labeling of the edges.

Now we state the main result of this second half of the paper. Its proof is almost an adaptation of the proof of Theorem 4.2 in [2] but care must be taken when, following a maximal chain, we move from $\mathrm{W}^{\mathrm{I}_{h}}$ to $\mathrm{W}^{\mathrm{I}_{h-1}}, h=n, \ldots, 2$.

Theorem 9.1. Let $(\mathrm{W}, \mathrm{S})$ be a Coxeter group, $\mathrm{I}_{1}, \ldots, \mathrm{I}_{n}$ be subsets of $\mathrm{S}$ and let $\Omega=\left(\mathrm{I}_{1}, \ldots, \mathrm{I}_{n}\right)$. Then any closed interval $[\tau, \sigma] \subset \mathrm{W}^{\Omega}$ is lexicographically shellable.

Proof. We show that the labeling described above is an L-labeling; so our previous notations will be in force throughout the proof.

(L1) is easily verified since at each step the element we remove from $\alpha$ is uniquely determined.

It suffices to verify (L2) only for the entire interval $[\tau, \sigma]$. Indeed let $([u, v], \sigma=$ $\left.y_{0}>y_{1}>\cdots>y_{r}=v\right)$ be a rooted interval in $[\tau, \sigma]$ and suppose $u \in \mathrm{W}^{\mathrm{I}_{h}}$, $v \in \mathrm{W}^{\mathrm{I}_{k}}, h \leq k$. Following $y_{0}>y_{1}>\cdots>y_{r}$ we produce a uniquely determined reduced expression $v=s_{1}^{\prime} s_{2}^{\prime} \cdots s_{p}^{\prime}$. The labeling of $[u, v]$ as a rooted interval of $[\tau, \sigma]$ is then equivalent to the labeling of $[u, v]$ directly obtained starting with the reduced expression $v=s_{1}^{\prime} s_{2}^{\prime} \cdots s_{p}^{\prime}$, i.e. considering the word $\alpha^{\prime}=$ $s_{1}^{\prime} s_{2}^{\prime} \cdots s_{p}^{\prime} \square s_{r_{n+1-h}+2} \cdots s_{r_{n+2-h}} \square s_{r_{n+2-h}+2} \cdots$.

Now we prove that at most one maximal chain in $[\tau, \sigma]$ has increasing label. Use induction on $t=$ length of the maximal chains of $[\tau, \sigma]$. If $t=1$ our claim 
is clear. If $\Omega=\left(\mathrm{I}_{1}\right)$, i.e. $n=1$, then our labeling reduces to the one in [2] and hence, being an L-labeling, it verifies (L2). So suppose $t>1, n>1$ and let

$$
\begin{gathered}
\mathbf{c}: \sigma=x_{0}>x_{1}>\cdots>x_{t_{1}} \triangleright x_{t_{1}+1}>\cdots>x_{t_{2}} \triangleright x_{t_{2}+1}>\cdots>x_{t}=\tau \\
\mathbf{c}^{\prime}: \sigma=x_{0}^{\prime}>x_{1}^{\prime}>\cdots>x_{t_{1}^{\prime}}^{\prime} \triangleright x_{t_{1}^{\prime}+1}^{\prime}>\cdots>x_{t_{2}^{\prime}}^{\prime} \triangleright x_{t_{2}^{\prime}+1}^{\prime}>\cdots>x_{t}^{\prime}=\tau
\end{gathered}
$$

be two maximal chains with increasing labels, say $\lambda(\mathbf{c})=\left(i_{1}, \ldots, i_{t}\right), \lambda\left(\mathbf{c}^{\prime}\right)=$ $\left(j_{1}, \ldots, j_{t}\right)$. By construction of the labeling this means

$$
\begin{aligned}
& \tau=s_{1} \cdots \hat{s}_{i_{1}} \cdots \hat{s}_{i_{2}} \cdots \hat{s}_{i_{t}} \cdots s_{r_{n}} \\
& \tau=s_{1} \cdots \hat{s}_{j_{1}} \cdots \hat{s}_{j_{2}} \cdots \hat{s}_{j_{t}} \cdots s_{r_{n}}
\end{aligned}
$$

We want to show $i_{t}=j_{t}$. Suppose $j_{t}>i_{t}$. From $\tau \in \mathrm{W}^{\mathrm{I}_{1}} \hookrightarrow \mathrm{W}^{\Omega}$ we have $j_{t}>i_{t} \geq r_{n-1}+1=$ the position of the last box in $\alpha$. Consider now $u=$ $s_{r_{n}} s_{r_{n}-1} \cdots s_{j_{t}+1} s_{j_{t}} s_{j_{t}+1} \cdots s_{r_{n}-1} s_{r_{n}} \in \mathrm{T}$, then

$$
x_{t-1}^{\prime}=x_{t}^{\prime} u=s_{1} \cdots \hat{s}_{i_{1}} \cdots \hat{s}_{i_{2}} \cdots \hat{s}_{i_{t}} \cdots \hat{s}_{j_{t}} \cdots s_{r_{n}}
$$

and so $\operatorname{len}\left(x_{t-1}^{\prime}\right) \leq \operatorname{len}\left(x_{t}^{\prime}\right)-1$. But this is impossible since $x_{t-1}^{\prime}>x_{t}^{\prime}$. Hence $j_{t} \leq i_{t}$ and, by symmetry, $i_{t}=j_{t}$. So $x_{t-1}^{\prime}=x_{t-1}$ and we can use induction on $\left[x_{t-1}, \sigma\right]$ to derive $\mathbf{c}=\mathbf{c}^{\prime}$.

Now let

$$
\mathbf{c}_{0}: \sigma=x_{0}>x_{1}>\cdots>x_{t_{1}} \triangleright x_{t_{1}+1}>\cdots>x_{t}=\tau
$$

be the chain such that $\lambda\left(\mathbf{c}_{0}\right)=\left(\lambda_{1}, \ldots, \lambda_{t}\right)$ is minimum in the lexicographic order. We claim that $\lambda\left(\mathbf{c}_{0}\right)$ is increasing. Suppose otherwise and let $1 \leq i \leq t-1$ be the least integer such that $\lambda_{i}>\lambda_{i+1}$.

Notice that if $x_{i+1}, x_{i-1}$ are contained in $\mathrm{W}_{\mathrm{I}_{h}} \hookrightarrow \mathrm{W}^{\Omega}$ for some $h$ then, reducing to the case $\mathrm{W}^{\Omega}=\left(\mathrm{I}_{h}\right)$ we can use the result of Björner and Wachs to obtain a contradiction.

Suppose $x_{i+1} \in \mathrm{W}^{\mathrm{I}_{h-1}}, x_{i-1} \in \mathrm{W}^{\mathrm{I}_{h}}$ for some $h$. No generality is lost if we suppose $h=n$, indeed if $h<n$ we can consider the interval $[\sigma, \bar{\tau}]$ where $\bar{\tau}$ is maximal such that $\bar{\tau} \in \mathbf{c} \cap \mathrm{W}^{\mathrm{I}_{h}}$. Now if $x_{i} \in \mathrm{W}^{\mathrm{I}_{n}}$ then $x_{i+1} \triangleleft x_{i}$, hence $\lambda_{i+1}=r_{1}+1>\lambda_{i}$. A contradiction with the assumption that $\lambda_{i}>\lambda_{i+1}$. So $x_{i} \in \mathrm{W}^{\mathrm{I}_{n-1}}$ and $x_{i-1} \triangleright x_{i}, \lambda_{i}=r_{1}+1$.

Notice that $\lambda_{j}<r_{1}+1$ for any $j<i$, hence we have the following reduced expressions:

$$
\begin{aligned}
x_{i-1} & =s_{1} \cdots \hat{s}_{\lambda_{1}} \cdots \hat{s}_{\lambda_{2}} \cdots \hat{s}_{\lambda_{i-1}} \cdots s_{r_{1}} & \in & \mathrm{W}_{\overline{I_{n}}}^{\mathrm{I}_{n}} \\
x_{i} & =s_{1} \cdots \hat{s}_{\lambda_{1}} \cdots \hat{s}_{\lambda_{2}} \cdots \hat{s}_{\lambda_{i-1}} \cdots s_{r_{1}} s_{r_{1}+2} \cdots s_{r_{2}} & \in & \mathrm{W}_{+}^{\mathrm{I}_{n-1}} \\
x_{i+1} & =s_{1} \cdots \hat{s}_{\mu_{1}} \cdots \hat{s}_{\mu_{2}} \cdots \hat{s}_{\mu_{i}} \cdots s_{r_{1}} s_{r_{1}+2} \cdots s_{r_{2}} & \in & \mathrm{W}^{\mathrm{I}_{n-1}}
\end{aligned}
$$

where $\left\{\mu_{1}<\mu_{2}<\cdots<\mu_{i}\right\}=\left\{\lambda_{1}, \lambda_{2}, \ldots, \lambda_{i-1}, \lambda_{i+1}\right\}$. Note that $\mu_{i} \leq r_{i}$ since $\lambda_{i+1}<\lambda_{i}=r_{1}+1$. Then by Lemma 7.4 we have $x_{i+1} \in \mathrm{W}_{+}^{\mathrm{I}_{n-1}}$ and so $x_{i+1}=\bar{x}_{i} w_{n}$ where

$$
\bar{x}_{i}=s_{1} \cdots \hat{s}_{\mu_{1}} \cdots \hat{s}_{\mu_{2}} \cdots \hat{s}_{\mu_{i}} \cdots s_{r_{1}} \in \mathrm{W}_{-}^{\mathrm{I}_{n}} .
$$


This suffices to costruct the chain (see Figure 8)

$$
\mathbf{c}_{1}: \sigma=x_{0}>\cdots>x_{i-1}>\bar{x}_{i} \triangleright x_{i+1}>\cdots>x_{t}=\sigma
$$

with label $\lambda\left(\mathbf{c}_{1}\right)=\left(\lambda_{1}, \ldots, \lambda_{i-1}, \lambda_{i+1}, r_{1}+1=\lambda_{i}, \lambda_{i+2}, \ldots, \lambda_{t}\right)$ that is lexicographically strictly less than $\lambda\left(\mathbf{c}_{0}\right)$. This contradicts the choice of $\mathbf{c}_{0}$, so $\lambda\left(\mathbf{c}_{0}\right)$ must be increasing.

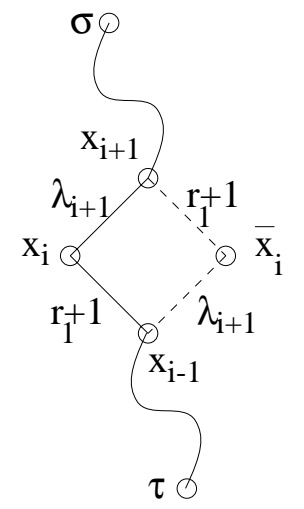

Figure 8. The chains in the proof of Theorem 9.1.

We must still check the case $x_{i-1} \in \mathrm{W}^{\mathrm{I}_{h+1}}, x_{i} \in \mathrm{W}^{\mathrm{I}_{h}}, x_{i+1} \in \mathrm{W}^{\mathrm{I}_{h-1}}$. But this is ruled out by our assumption that $\lambda_{i}>\lambda_{i+1}$ as in this case we have $\lambda_{i}=r_{n-h}<r_{n-h+1}=\lambda_{i+1}$

\section{References}

[1] A. Björner, Shellable and Cohen-Macaulay partially ordered sets, Trans. Amer. Math. Soc. 260 (1980), no. 1, 159-183.

[2] A. Björner, M. Wachs, Bruhat order of Coxeter groups and shellability, Adv. in Math. 43 (1982), no. 1, 87-100.

[3] N. Bourbaki, Eléments de mathématique. Fasc. XXXIV. Groupes et algèbres de Lie. Chapitres IV, V, VI, Hermann, Paris, 1968, Actualités Scientifiques et Industrielles, No. 1337.

[4] R. Chirivì, A note on jeu de taquin, Atti Accad. Naz. Lincei Cl. Sci. Fis. Mat. Natur. Rend. Lincei (9) Mat. Appl. 10 (1999), no. 4, 219-228.

[5] R. Chirivì, LS algebras and application to Schubert varieties, Transform. Groups 5 (2000), no. 3, 245-264.

[6] V. V. Deodhar, Some characterizations of Bruhat ordering on a Coxeter group and determination of the relative Möbius function, Invent. Math. 39 (1977), no. $2,187-198$. 
[7] N. Gonciulea, V. Lakshmibai, Degenerations of flag and Schubert varieties to toric varieties, Transform. Groups. 1 (1996), no. 3, 215-248.

[8] H. Hiller, The geometry of Coxeter groups, Research Notes in Mathematics 54, Pitmann Advanced Publishing Program, Boston, Mass., 1982.

[9] J. E. Humphreys, Reflection groups and Coxeter groups, Cambridge University Press, Cambridge, 1990.

[10] C. Huneke, V. Lakshmibai, A characterization of Kempf varieties by means of standard monomials and the geometric consequences, J. Algebra 94 (1985), no. $1,52-105$.

[11] V. G. Kac, Infinite dimensional Lie algebras, third ed., Cambridge University Press, Cambridge, 1990.

[12] G. R. Kempf, A. Ramanathan, Multi-cones over Schubert varieties, Invent. Math. 87 (1987), no. 2, 353-363.

[13] V. Lakshmibai, C. Musili, C.S. Seshadri, Geometry of G/P , Bull. Amer. Math. Soc. (N.S.) 1 (1979), no. 2, 432-435.

[14] V. Lakshmibai, C. Musili, C.S. Seshadri, Geometry of G/P . III. Standard monomial theory for a quasi minuscule P, Proc. Indian. Acad. Sci. Sect. A Math. Sci. 88 (1979), no. 3, 93-177.

[15] V. Lakshmibai, C. Musili, C.S. Seshadri, Geometry of G/P . IV. Standard monomial theory for classical types, Proc. Indian. Acad. Sci. Sect. A Math. Sci. $8 \mathbf{8}$ (1979),no. 4, 279-362.

[16] V. Lakshmibai, P. Littelmann, P. Magyar, Standard monomial theory and applications. Representation theories and algebraic geometry (Montreal, PQ, 1997), Kluwer Acad. Publ., Dordrecht, 1998, Notes by R.W.T. Yu, pp. 319-364.

[17] A. Lascoux, M.-P. Schützenberger, Le monoï d plaxique, Non-commutative structures in algebra and geometric combinatorics (Naples, 1978), CNR, Rome, 1981, pp. 129-156.

[18] P. Littelmann, Paths and root operators in representation theory, Ann. of Math. (2) 142 (1995), no. 3, 499-525.

[19] P. Littelmann, A plactic algebra for semisimple Lie algebras, Adv. in Math. 124 (1996), no. 2, 312-331.

[20] P. Littelmann, Contracting modules and standard monomial theory for symmetrizable Kac-Moody algebras, J. Amer. Math. Soc. 11 (1998), no. 3, 551-567.

[21] G. A. Reisner, Cohen-Macaulay quotients of polynomial rings, Adv. in Math. 21 (1976), no. 1, 30-49.

[22] C. S. Seshadri, Geometry of G/P. I. Theory of standard monomials for minuscule representations, C.P. Ramanujan - a tribute, Springer, Berlin, 1978,pp. 207-239.

[23] R. P. Stanley, Cohen-Macaulay rings and costructible polytopes, Bull. Amer. Math. Soc. 81 (1975), 133-135.

[24] D.-N. Verma, Möbius inversion for Bruhat ordering on a Weyl group, Ann. Sci. École Norm. Sup. (4) 4 (1971), 393-398.

[25] D.-N. Verma, A strengthening of the exchange property of Coxeter groups, preprint, 1972 . 
Rocco Chirivì

Dipartimento di Matematica

"Guido Castelnuovo"

Università di Roma "La Sapienza"

Piazzale Aldo Moro 5

I-00185 Roma

Italy

e-mail: chirivi@mat.uniroma1.it

(Received: June 23, 2000) 